\title{
Identification of MRP4/ABCC4 as a Target for Reducing the Proliferation of Pancreatic Ductal Adenocarcinoma Cells by Modulating the cAMP Efflux ${ }^{[}$
}

\author{
Alejandro Carozzo, ${ }^{1}$ Agustín Yaneff, ${ }^{1}$ Natalia Gómez, Nicolás Di Siervi, Ana Sahores, \\ Federico Diez, Alejandra I. Attorresi, Ángela Rodríguez-González, Federico Monczor, \\ Natalia Fernández, Martín Abba, Carina Shayo, and Carlos Davio
}

Instituto de Investigaciones Farmacológicas (ININFA-UBA-CONICET), Facultad de Farmacia y Bioquímica, Universidad de Buenos Aires, Buenos Aires, Argentina (A.C., A.Y., N.G., N.D.S., A.S., F.D., F.M., N.F., C.D.); IBioBA MPSP - Instituto de Investigaciones en Biomedicina de Buenos Aires, CONICET, Instituto Partner de la Sociedad Max Planck, Buenos Aires, Argentina (A.I.A.); Instituto de Biología y Medicina Experimental (IBYME-CONICET), Buenos Aires, Argentina (Á.R.-G., C.S.); and Centro de Investigaciones Inmunológicas Básicas y Aplicadas, Facultad de Ciencias Médicas, Universidad Nacional de La Plata, Buenos Aires, Argentina (M.A.)

Received December 5, 2018; accepted April 17, 2019

\section{ABSTRACT}

Pancreatic cancer is one of the most lethal types of tumors with no effective therapy available; is currently the third leading cause of cancer in developed countries; and is predicted to become the second deadliest cancer in the United States by 2030. Due to the marginal benefits of current standard chemotherapy, the identification of new therapeutic targets is greatly required. Considering that CAMP pathway is commonly activated in pancreatic ductal adenocarcinoma (PDAC) and its premalignant lesions, we aim to investigate the multidrug resistance-associated protein 4 (MRP4)-dependent cAMP extrusion process as a cause of increased cell proliferation in human PDAC cell lines. Our results from in silico analysis indicate that MRP4 expression may influence PDAC patient outcome; thus, high MRP4 levels could be indicators of poor survival. In addition, we performed in vitro experiments and identified an association between higher MRP4 expression levels and more undifferentiated and malignant models of PDAC and CAMP extrusion capacity. We studied the antiproliferative effect and the overall cAMP response of three MRP4 inhibitors, probenecid, MK571, and ceefourin-1 in PDAC in vitro models. Moreover, MRP4-specific silencing in PANC-1 cells reduced cell proliferation $(P<0.05)$, whereas MRP4 overexpression in BxPC-3 cells significantly incremented their growth rate in culture $(P<0.05)$. MRP4 pharmacological inhibition or silencing abrogated cell proliferation through the activation of the cAMP/Epac/Rap1 signaling pathway. Also, extracellular CAMP reverted the antiproliferative effect of MRP4 blockade. Our data highlight the MRP4-dependent cAMP extrusion process as a key participant in cell proliferation, indicating that MRP4 could be an exploitable therapeutic target for PDAC.

\section{SIGNIFICANCE STATEMENT}

ABCC4/MRP4 is the main transporter responsible for cAMP efflux. In this work, we show that MRP4 expression may influence PDAC patient outcome and identify an association between higher MRP4 expression levels and more undifferentiated and malignant in vitro models of PDAC. Findings prove the involvement of MRP4 in PDAC cell proliferation through a novel extracellular cAMP mitogenic pathway and further support MRP4 inhibition as a promising therapeutic strategy for PDAC treatment.
The authors declare no competing financial interests.

This work was supported by Universidad de Buenos Aires [Grant UBACYT20020130100624BA], Agencia Nacional de Promoción Científica y Tecnológica [Grants PICT-2013-2050, PICT-2016-1821, and PICT-2015-2996], and Consejo Nacional de Investigaciones Científicas y Técnicas [Grant PIP-112-201201-00562].

${ }^{1}$ A.C. and A.Y. contributed equally.

https://doi.org/10.1124/mol.118.115444.

S This article has supplemental material available at molpharm. aspetjournals.org.

\section{Introduction}

The cAMP pathway is one of the most studied signaling cascades in cellular biology and particularly related to cancer. It has been demonstrated that aberrant cyclic nucleotide signaling may play an important role in several types of neoplasias (Fajardo et al., 2014). Briefly, in this signaling pathway, the production of cAMP by adenylyl cyclase is stimulated or inhibited by the activation of $G$ proteincoupled receptors coupled to a $\mathrm{G} \alpha$ stimulatory subunit or to a G $\alpha$ inhibitory subunit, respectively. Once synthesized,

ABBREVIATIONS: AMPCP, Adenosine 5'-[alpha,beta-methylene]diphosphate; AUC, area under the curve; CSC, cancer stemlike cells; e-cAMP, extracellular CAMP; EPAC, exchange proteins activated by cAMP; FDA, Food and Drug Administration; FRET, Föster resonance energy transfer; i-cAMP, intracellular cAMP; MRP4, multidrug resistance-associated protein 4; PDAC, pancreatic ductal adenocarcinoma; PDE, phosphodiesterase; PKA, protein kinase A; PKI, PKA inhibitor peptide; $\mathrm{PPCR}$, quantitative reverse-transcription polymerase chain reaction; RBP, radiobinding protein; shRNA, short hairpin RNA; WB, Western blot. 
cAMP acts on its main effectors: protein kinase A (PKA), exchange proteins activated by cAMP (EPAC), cyclic nucleotidegated ion channels, or Popeye domain-containing proteins. Signal attenuation of this second messenger occurs by its degradation by phosphodiesterases (PDE), compartmentalization, desensitization of $\mathrm{G}$ protein-coupled receptors, and/or extrusion to the extracellular compartment mainly mediated by the multidrug resistance-associated protein 4 (MRP4)/ABCC4 (Beavo and Brunton, 2002; Sassi et al., 2009).

Pancreatic ductal adenocarcinoma (PDAC) is one of the most lethal types of tumors and leading therapeutic challenges for modern oncology (Rahib et al., 2014). Despite the recent improvement in survival using FOLFIRINOX (folinic acid, fluorouracil, irinotecan, and oxaliplatin) or nabpaclitaxel plus gemcitabine, the 5-year survival rate remains below 5\%-8\% (Siegel et al., 2017). Therefore, there is a great clinical need to identify key molecular and cellular regulators of PDAC tumor progression and invasion to develop alternative therapeutic strategies. The carcinogenic process of pancreatic cancer accounts for an accumulation of mutations and genetic lesions in signaling pathways linked to cell proliferation, motility, and survival. In the last few decades, several mutations leading to activation of oncogenes, such as KRAS, $G N A S$, and $B R A F$, and inhibition of tumor suppressor genes, such as p16/CDNKN2A, TP53, SMAD4/DPC4, and BRCA2, have been identified (Cancer Genome Atlas Research Network, 2017). These mutations are present in the earliest stages and lowest-grade premalignant lesions of PDAC, suggesting that they could be essential for the development and/or progression of the disease, and therefore conceivably therapeutic targets (Kanda et al., 2012).

Interestingly, frequent activating mutations in GNAS, gene encoding for the $\mathrm{G} \alpha \mathrm{s}$ subunit, are not the only alterations in the canonical cAMP signaling pathway described in PDAC (Cancer Genome Atlas Research Network, 2017). Recent studies identified that $59 \%$ of the tested human PDAC samples exhibit an elevated $\mathrm{G} \alpha$ s expression compared with normal tissues, and $72 \%$ show an increment in the expression of phosphorylated substrates of PKA, regardless whether they have GNAS mutations (Furukawa et al., 2011; Wu et al., 2011; Kanda et al., 2012; O'Hayre et al., 2013). Also, MRP4 has shown to be highly expressed in pancreatic cancer tissues, and its silencing in PDAC cell lines reduces in vitro proliferation with a cell cycle arrest in G1 phase (Zhang et al., 2012). However, little is known about the mechanism involved in the antiproliferative effect of MRP4 inhibition, and there are limited studies of its potential as a therapeutic target in cancer. In this regard, it has been recently described that enhanced intracellular cAMP (i-cAMP) levels upregulate MRP4 expression through the activation of cAMP/Epac/Rap1 signaling pathway (Bröderdorf et al., 2014; Carozzo et al., 2015). In recent years, interest in the expression and activity of MRP4 has been awakened in cancer studies (Yaneff et al., 2017). Numerous publications describe the antiproliferative effect of MRP4 inhibition, its upregulation in several tumors, and its potential as a marker of poor prognosis (Wen et al., 2015; Yaneff et al., 2017).

Given that: 1) the cAMP pathway is frequently enhanced in PDAC, 2) intracellular cAMP enhancement is associated with inhibition of cell proliferation in this type of cancer (Boucher et al., 2001; Lorenz et al., 2008; Burdyga et al., 2013), and 3)
MRP4 mediates cAMP efflux, we hypothesized that increased MRP4 expression levels could generate an imbalance between the ratio of extracellular cAMP (e-cAMP) and i-cAMP that triggers a global proliferative response in PDAC cells. Thus, MRP4 inhibition would alter this imbalance and, consequently, impair cell proliferation. In the present work, we sought to determine the role of the MRP4-dependent cAMP extrusion process and its involvement in PDAC cell proliferation using human PDAC cell lines with different degrees of differentiation to propose MRP4 as an exploitable therapeutic target for this malignancy.

\section{Materials and Methods}

\section{In Silico Analysis of MRP4/ABCC4 Expression among PDAC Cases}

The prognostic value of MRP4/ABCC4 expression in patients with PDAC was evaluated using the TCGA-PAAD RNAseq pancreatic dataset obtained from the University of California (Santa Cruz) Xena resource (https://xena.ucsc.edu). A group of 178 patients with pancreatic cancer and follow-up data was classified into low or high MRP4/ABCC4 mRNA expression levels according to the StepMiner one-step algorithm (Sahoo et al., 2007). These groups were then compared based on the recurrence-free survival (Kaplan-Meier curves and log-rank test) using the Survival $R$ package (Therneau and Grambsch, 2000).

\section{Cell Lines}

Human PDAC cell lines, PANC-1 (CRL-1469), BxPC-3 (CRL1687), and HPAF-II (CRL-1997), and human embryonic kidney cell line, HEK293T (CRL-3216), were obtained from the American Type Culture Collection and cultured in Dulbecco's modified Eagle's medium (PANC-1, HEK293T) or RPMI 1640 (BxPC-3, HPAF-II) media (Sigma-Aldrich) supplemented with 10\% FBS (Natocor, Argentina) and $50 \mu \mathrm{g} / \mathrm{ml}$ gentamycin (Sigma-Aldrich) at $37^{\circ} \mathrm{C}$ in a humidified $5 \% \mathrm{CO}_{2}$ atmosphere. Cells were routinely checked for mycoplasma contamination using the PlasmoTest (InvivoGen). All experiments were performed using cells in the exponential growth phase and when cell viability was above $95 \%$, as determined by trypan blue (Sigma-Aldrich) assay.

\section{Cell Cycle Synchronization at G0/G1 Phase}

Cell cycle synchronization was achieved by serum-starved for 24 hours at $37^{\circ} \mathrm{C}$ in a humidified $5 \% \mathrm{CO}_{2}$ atmosphere, and cells were relieved into cell cycle by adding $10 \% \mathrm{FBS}$.

\section{Western Blotting}

A total of $5 \times 10^{5}$ cells/well was seeded in a 12 -well plate in its respective media supplemented with $10 \% \mathrm{FBS}$. After cell attachment, cells were synchronized and further incubated for 24 hours in fresh culture media with $10 \%$ FBS. In all cases, whole-cell extracts were obtained using radioimmunoprecipitation assay lysis buffer (Thermo Fisher Scientific) and resolved by 8\% SDS-PAGE loading equal amounts of protein $(20 \mu \mathrm{g})$ per lane. Blots were probed concomitantly as the nitrocellulose membranes were cut in half to allow two different primary antibodies to be used. The top half was used to detect MRP4 protein (M4I-10, MA1-35681; $3 \mu \mathrm{g} / \mathrm{ml}$; Thermo Scientific), and the bottom half (less than $70 \mathrm{kDa}$ ) was used to detect the normalization protein, $\beta$-tubulin (H-300; $1 \mu \mathrm{g} / \mathrm{ml}$; Abcam), which was used to confirm equal loading and transfer.

In all cases, reactivity was developed using either anti-rat (sc-2006; Santa Cruz Biotechnology) or anti-rabbit (PI-1000; Vector Laboratories) antibodies linked to horseradish peroxidase and ECL Western Blotting Detection Reagent following the manufacturer's instructions 
(Amersham Biosciences). Immunoblots were quantified using Image $\mathrm{J}$ (Schneider et al., 2012). For quantification, the background value of the scanned gel was subtracted, and the relative abundance was determined according to MRP4 $=(\mathrm{MRP} 4) /(\beta$-tubulin $)$. Relative abundance was then normalized respective to the control group.

\section{Quantitative RT-PCR}

Cells were seeded in a six-well plate at $5 \times 10^{5}$ cells/well following 24-hour incubation in fresh culture media with $10 \% \mathrm{FBS}$ at $37^{\circ} \mathrm{C}$ in a humidified $5 \% \mathrm{CO}_{2}$ atmosphere. Total RNA was extracted using Quick-Zol reagent (Kalium Technologies) and reverse-transcribed using the High Capacity cDNA Reverse Transcription kit (Applied Biosystems) following the manufacturer's instructions. Quantitative reverse-transcription polymerase chain reaction (qPCR) was performed in triplicate using HOT FIREPol EvaGreen qPCR Mix Plus (Solis Biodyne) and a Rotor Gene Q detection system (Qiagen); cycling parameters were as follows: 15 seconds at $94^{\circ} \mathrm{C}, 20$ seconds at $60^{\circ} \mathrm{C}$, and 30 seconds at $72^{\circ} \mathrm{C}$ for 45 cycles. The following primers were used: hMRP4_FW: 5'-GGACAAAGACAACTGGTGTGCC-3', hMRP4_RV: $5^{\prime}$-AATGGTTAGCACGGTGCAGTGG-3', $\beta$-actin_FW: $5^{\prime}$-GGACTTCGAGCAAGAGATGG-3', and $\beta$-actin_RV: $5^{\prime}$-AGCACTGTGTTGGCGTACAG-3' . The relative MRP4 mRNA quantification was performed using the comparative $\Delta \Delta \mathrm{Ct}$ method, with $\beta$-actin as the housekeeping gene.

\section{cAMP Radiobinding Protein Assay}

In all cases, cells were seeded in 24 -well plates at a density of $1 \times 10^{5}$ cells/well, and, before starting each experiment, culture media was replaced with phenol red-free media (Sigma-Aldrich) without FBS. Cells were then exposed to various agents at different concentrations and time points, as indicated in the corresponding figure legends. No PDE inhibitors were used during cAMP accumulation assays. Following treatment, each well (i-cAMP) and well supernatant (e-cAMP) was extracted overnight with $95 \% \mathrm{v} / \mathrm{v}$ ethanol. Extracts were then evaporated, and residues were resuspended in radiobinding protein (RBP) buffer (50 mM Tris-HCl, $4 \mathrm{mM}$ EDTA, $\mathrm{pH} 7.4,0.1 \%$ bovine serum albumin). Blinding was applied to these samples as they were labeled with consecutive numbers and the corresponding measurements were performed without knowing which sample corresponded to each number. cAMP content was determined by a competitive $\mathrm{RBP}$ assay for PKA using $\left[{ }^{3} \mathrm{H}\right]$-cAMP, as previously described (Davio et al., 1995). Briefly, titrated PKA was incubated in equilibrium conditions $\left(2\right.$ hours, $\left.4^{\circ} \mathrm{C}\right)$ with different samples or cAMP standards (0.1-90 pmol) in the presence of $2 \mathrm{nM}\left[{ }^{3} \mathrm{H}\right]$-cAMP $(20.7 \mathrm{Ci} / \mathrm{mmol}$, NET1161250UC; PerkinElmer) in RBP buffer. The bound fraction was separated by carbon-dextran precipitation, followed by centrifugation $\left(2000 \mathrm{~g}, 15\right.$ minutes, $\left.4^{\circ} \mathrm{C}\right)$, and Optiphase HiSafe 3 scintillation cocktail (PerkinElmer) was added to each supernatant for counting in a Pharmacia Wallac 1410 counter. Sample cAMP concentrations were determined by interpolating from the displacement curves obtained from cAMP standards using Prism 6.0 (GraphPad Software). Duplicate samples of at least three independent experiments were analyzed. For concentration-response assays, we fit the pooled data from all experiments into a single equation. A fourth experiment was sometimes needed to properly adjust concentration-response equations.

\section{Transient Transfections}

All transient transfections were carried out as follows: cells at $80 \%$ confluence in a 24-well plate were incubated with $8 \mu \mathrm{l}$ K2 Multiplier reagent (Biontex K2 Transfection System) for 2 hours before adding $500 \mathrm{ng}$ either empty vector (control) or coding cDNA constructs with $1 \mu \mathrm{l} \mathrm{K} 2$ transfection reagent in a final volume of $600 \mu \mathrm{l}$ fresh culture media. All experiments involving transiently transfected cells were carried out 24 hours post-transfection, and culture media was replaced with fresh media.

\section{Generation of Stable MRP4-Silenced PANC-1 Cells}

Short Hairpin RNA Expression Plasmids. Oligonucleotides containing two different short hairpin RNA (shRNA) sequences targeting MRP4 (MRP4-shRNA1 and MRP4-shRNA2) and a scramble shRNA sequence noncomplementary to any human gene were designed according to the pSUPER.retro.puro Manual (OligoEngine) and previously validated (Sassi et al., 2008; Copsel et al., 2011). Oligonucleotides were cloned into the pSUPER.retro.puro vector (OligoEngine); their correct orientation after cloning was confirmed by DNA sequencing using the following primer: 5'-GGAAGCCTTGGCTTTTG-3' (Macrogen). MRP4-shRNA oligonucleotides contained a MRP4 mRNAspecific region, a hairpin loop region (bold), and a linker sequence for subcloning into the pSUPER vector (italic): MRP4-shRNA1 sequence $\left(5^{\prime}-3^{\prime}\right)$ sense: GATCCCCCAGTGTTCTTACACTTCCTTTCAAGAGAAGGAAGTGTAAGAACACTGTTTTTA; antisense: AGCTTAAAAACAGTGTTCTTACACTTCCTTCTCTTGAAAGGAAGTGTAAGAACACTGGGG; MRP4-shRNA2 sequence ( $\left.5^{\prime}-3^{\prime}\right)$ sense: GATCCCCGATGGTGCATGTGCAGGATTTCAAGAGAATCCTGCACATGCAGCATCTTTTTA; antisense: AGCTTAAAAAGATGGTGCATGTGCAGGATTCTCTTGAAATCCTGCACATGCAGCATCGGG; scramble-shRNA sequence $\left(5^{\prime}-3^{\prime}\right)$ sense: GATCCCCGAAACTGCTGACCGTTAATTTCAAGAGAATTAACGGTCAGCAGTTTCTTTTTA; antisense:AGCTTAAAAAGAAACTGCTGACCGTTAATTCTCTTGAAATTAACGGTCAGCAGTTTCGGG.

\section{Stable PANC-1 Cells Expressing shRNA Targeting MRP4}

Retroviruses were generated in HEK293T cells seeded in a 10-cm plate 1 day prior to calcium phosphate-mediated cotransfection with a sequence of either pSUPER-MRP4-shRNA or pSUPER-scrambleshRNA in combination with viral packaging expression plasmids (Addgene). Viral supernatants were collected 48 hours later and stored in $-80^{\circ} \mathrm{C}$ after clarification.

Stable expressing pSUPER-MRP4-shRNA1 (PANC-1 MRP4sh1), pSUPER-MRP4-shRNA2 (PANC-1 MRP4sh2), or pSUPER-scrambleshRNA (PANC-1 scramble) cells were obtained by transduction in PANC- 1 cells seeded at $1 \times 10^{6} /$ well in a six-well plate 24 hours before adding $1 \mathrm{ml}$ viral supernatant together with $10 \mu \mathrm{g} / \mathrm{ml}$ polybrene. Forty-eight hours after infection, clonal selection was carried out in the presence of $3 \mu \mathrm{g} / \mathrm{ml}$ puromycin (Sigma-Aldrich). Clones were tested for MRP4 expression by qPCR and Western blot (WB). Specific clones presenting lower MRP4 expression were chosen for further experiments and maintained in media containing $1 \mu \mathrm{g} / \mathrm{ml}$ puromycin.

\section{Stable BxPC-3 Cells Overexpressing MRP4}

The pcDNA3.1/hygro (-) MRP4 and empty vector were provided by M. Grube from Institut fur Pharmakologie, Universitat Medizin Greifswald (Rius et al., 2003). Stable expressing pcDNA3.1/hygro (-) MRP4 (BxPC-3 MRP4) and empty (BxPC-3 mock) cells were obtained by transfection of BxPC-3 cells seeded at $1 \times 10^{6} /$ well in a six-well plate 24 hours before adding the corresponding DNA vector $(1 \mu \mathrm{g})$ together with $2 \mu \mathrm{l} \mathrm{K} 2$ Multiplier reagent. Twenty-four hours after transfection, cells were seeded in the presence of $200 \mu \mathrm{g} / \mathrm{ml}$ hygromycin B (SelleckChem) for 2 weeks, and clonal selection was carried out in 96-well plates for another 2 weeks. Finally, clones were tested for MRP4 expression by qPCR and WB. Both BxPC-3 mock and BxPC-3 MRP4 were maintained in media containing $100 \mu \mathrm{g} / \mathrm{ml}$ hygromycin B.

\section{Stable BxPC-3 Cells Overexpressing mTurquoise2-EPAC- cp173Venus-Venus (Epac-S ${ }^{\mathrm{H187}}$ )}

Stable expressing pcDNA3.1/Zeo(+)-mTurquoise2-EPAC-cp173VenusVenus (Epac-S ${ }^{\mathrm{H} 187}$ ) (BxPC-3 Epac-S ${ }^{\mathrm{H} 187}$ ) and empty (BxPC-3 mock) cells were obtained by transfection of BxPC-3 cells seeded at $1 \times 10^{6} \%$ well in a six-well plate 24 hours before adding the corresponding DNA vector $(1 \mu \mathrm{g})$ together with $2 \mu \mathrm{l} \mathrm{K} 2$ transfecting reagent (Biontex). Twenty-four hours after transfection, cells were seeded in the presence of $25 \mu \mathrm{g} / \mathrm{ml}$ zeocine (InvivoGen) for 2 weeks, and 
clonal selection was carried out in 96 -well plates for another 2 weeks. Finally, clones were tested for Epac- $\mathrm{S}^{\mathrm{H} 187}$ by fluorescence spectra $(450-650 \mathrm{~nm})$ measurements in a FlexStation 3 Multi-Mode Microplate Reader (Molecular Devices) with excitation at $430 \mathrm{~nm}$. The BxPC-3 Epac-S ${ }^{\mathrm{H} 187}$ clone with higher fluorescence emission was chosen for further experiments. Both BxPC-3 mock and BxPC-3 Epac- $\mathrm{S}^{\mathrm{H} 187}$ were maintained in media containing $25 \mu \mathrm{g} / \mathrm{ml}$ zeocine.

\section{Föster Resonance Energy Transfer Time Course of cAMP Intracellular Levels with Confocal Microscopy}

PANC-1 cells were transfected with mTurquoise2-EPACcp173Venus-Venus (Epac-S ${ }^{\mathrm{H} 187}$ ) (Cell Biophysics \& Imaging Group at Netherlands Cancer Institute) and seeded $4 \times 10^{4}$ cells/well in 24-well glass-bottom dishes. After 48 hours, cell imaging was performed on an inverted LSM 710 (ZEISS) microscope equipped with an automated stage, an incubation chamber, and the Definite Focus system. Data acquisition was performed with ZEN Black 2011 software (Carl Zeiss Microscopy). Images were acquired with a C-Apochromat $40 \times / 1.2$ water immersion and temperature-corrected objective lens at $1024 \times 1024$ pixels, 16 bits, pixel dwell time 3.15 microseconds, with open pinhole $(600 \mu \mathrm{m})$. The emission spectra of the fluorescent proteins mTurquoise 2 and Venus were determined using a 405-nm laser for the excitation and $\lambda$ mode for the detection using a 32-channel QUASAR detector arranged with 9.7-nm bandwidth channels.

For FRET experiments, cells were illuminated with a $30-\mathrm{mW}$, 405 -nm diode laser at $2 \%$ laser power $(550-650 \mathrm{~nm}$ gain $)$ and a $405-\mathrm{nm}$ dichroic mirror; emission was collected at 413- to 723-nm wavelengths every 15 seconds for 13 minutes. The saturation level was verified for each image. For the reference spectra and the experiments, phenol red-free Dulbecco's modified Eagle's medium (Sigma-Aldrich) was used, and imaging was performed at $37^{\circ} \mathrm{C}$ and $5 \% \mathrm{CO}_{2}$. Approximately 3 minutes after starting the experiment, $25 \mu \mathrm{M}$ MK571 (SigmaAldrich) or vehicle (0.01\% DMSO; Carlo Erba), followed by $0.5 \mu \mathrm{M}$ isoproterenol (Tocris) 5 minutes after, was added. For lambda FRET experiments, the linear unmixing tool of ZEN Black 2011 software was used to obtain an image for each fluorophore using the reference spectra. Dead cells fluoresced significantly more than live cells and were not considered for the analysis. Cell viability was confirmed by phase-contrast microscopy immediately after the i-cAMP measurement. Image quantification was performed with Fiji software (Schindelin et al., 2012). After background subtraction, FRET and donor intensities were measured for single cells for each time point (six to eight cells in each treatment, $n=3$ ). The FRET/donor ratio intensity was calculated and normalized to basal levels (before stimulation).

\section{FRET Time Course of cAMP Intracellular Levels with Flex Station 3}

Stable BxPC-3 cells overexpressing mTurquoise2-EPACcp173Venus-Venus (Epac-S ${ }^{\mathrm{H} 187}$ ) or pcDNA3.1/zeo (-) (empty vector) were seeded in 96 -well plates at a density of $1 \times 10^{5}$ cells/well. Before starting each experiment, cells were washed with $0.9 \% \mathrm{NaCl}$ twice, and $100 \mu \mathrm{l}$ of this solution was added to each well before placing the plate in a FlexStation 3 at $37^{\circ} \mathrm{C}$. To determine i-cAMP response, we measured the baseline fluorescence signal detected at 470 (donor) and $510 \mathrm{~nm}$ (FRET) emission with excitation at $430 \mathrm{~nm}$. Using the on-board pipettor, we added $50 \mu \mathrm{l} 3 \times$ inhibitor after 40 seconds and then monitored the signal every 20 seconds for a total of 420 seconds, both in stable Epac- $\mathrm{S}^{\mathrm{H} 187}$ and pcDNA3.1/zeo (-) overexpressing cells. Different concentrations of probenecid, MK571, and ceefourin-1 were added by automated fluid transfer. After background subtraction [BxPC-3 pcDNA3.1/zeo (-) treated with each inhibitor concentration], FRET and donor intensities were calculated in each well for each time point. The FRET/donor ratio intensities were calculated and normalized to basal levels-before stimulation- $\left(\mathrm{R} / \mathrm{R}_{0}\right)$ for each time point.
Triplicate samples of at least three independent experiments $(n=3)$ were analyzed. For concentration-response assays, we fit the pooled data from all experiments into a single equation.

\section{Cell Proliferation and Viability Assay}

For endpoint experiments, cells growing in exponential phase were seeded at $1 \times 10^{4}$ cells/well in a 12 -well plate. After synchronization, cells were incubated for 72 hours in fresh culture media with $10 \%$ FBS in the conditions indicated in each figure legend. For kinetic studies, cells growing in exponential phase were seeded at $1.5 \times 10^{4}$ cells/well in a 12-well plate. After synchronization, cells were incubated for different time points in fresh culture media with $10 \%$ FBS in the conditions indicated in each figure legend. In all cases, cells were harvested at the end of the experiment, and the total viable and nonviable cell number per well were determined by counting in a hemocytometer chamber after incubation with $0.4 \%$ trypan blue. Blinding was applied during sample preparation and analysis, as described above for the RBP assays. Triplicate samples of three independent experiments $(n=3)$ were analyzed.

\section{Cell Cycle Analysis}

Cells were seeded at $5 \times 10^{5}$ cells/well in a six-well plate, synchronized, and incubated for 72 hours in fresh culture media with $10 \%$ FBS, as indicated in each figure legend. After treatment, cells were harvested and washed with ice-cold PBS, fixed by addition of $70 \%$ $\mathrm{v} / \mathrm{v}$ ethanol, and stored at $-20^{\circ} \mathrm{C}$ for 24 hours. Cell suspensions were washed with ice-cold PBS and incubated with RNase A $(100 \mu \mathrm{g} / \mathrm{ml}$ in PBS; Sigma-Aldrich) at room temperature for 15 minutes. Propidium iodide (Sigma-Aldrich) was then added to a final concentration of $20 \mu \mathrm{g} / \mathrm{ml}$ and incubated in the dark for 30 minutes. Cell cycle phase distributions were analyzed by FACScan flow cytometer (BecktonDickinson). Blinding was applied during sample preparation and analysis, as described above for the RBP assays. Data from duplicates of three independent experiments $(n=3)$ were analyzed using ModFit software (Verity Software House) to determine the fractions of cells in the G0/G1, S, and G2/M phases from cell cycle distribution.

\section{Statistical Analysis}

In all cases, minimum sample size to ensure an adequate statistical power $(>0.95)$ was determined with $\mathrm{G}^{*}$ Power 3.1 software (Faul et al., 2007, 2009) using S.D. values of emerging experimental data as input. All statistical analyses were performed using Prism 6.0 (GraphPad Software). All intergroup statistical comparisons were specified before the experiments started, and all specific intergroup comparisons were explicitly reported. Experimental results are expressed as mean \pm S.D. of at least three independent experiments $(n=3)$. Paired samples comparisons were used for data analysis of experiments where a single cell line was assessed and unpaired samples comparisons were used in experiments where more than one cell line was assessed. All $t$ tests are two-sided with $\alpha=5 \%$. All analyses of variance are one- or two-way with post hoc $p$-adjustments using Bonferroni correction. Values of $P<0.05$ were considered statistically significant.

\section{Results}

MRP4 Expression May Influence PDAC Patients' Outcome. As it has been previously described, higher MRP4 expression levels were found in human pancreatic tumor samples compared with normal tissue, indicating an association between MRP4 levels and this type of cancer (Zhang et al., 2012) To assess the significance of MRP4 protein levels in PDAC prognosis, we determined whether MRP4 mRNA expression could be linked to patient overall survival. MRP4 gene expression profiles from 178 PDAC patients were obtained from TCGA-PAAD dataset and subsequently grouped according to 

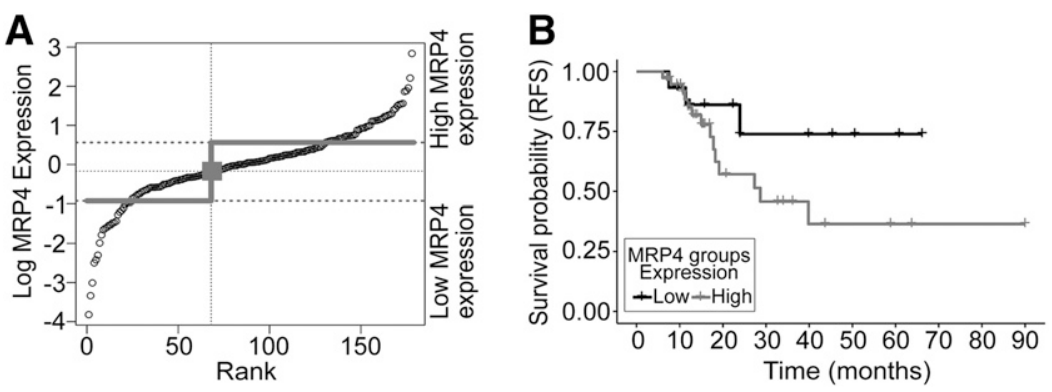

Fig. 1. MRP4 expression may influence patient outcome. (A) MRP4 gene expression profile from 178 PDAC cancer patients obtained from TCGA-PAAD RNAseq dataset (UCSC Xena), classified into two groups according to low (24/178) or high (48/178) MRP4 mRNA levels. (B) Prognostic value of MRP4 expression in grouped patients. Statistics: the comparison was based on recurrence-free survival (Kaplan-Meier curves and log-rank test; $P=0.15$ ). low or high MRP4 expression levels based on the cutoff value provided by the StepMiner algorithm (Fig. 1A). As shown in Fig. 1B, Kaplan-Meier analysis revealed that the subgroup of patients with high MRP4 expression is associated with a trend toward shorter recurrence-free survival compared with those with low expression $(P=0.15)$, despite the short follow-up times of patients available at TCGA-PAAD dataset (median recurrence-free survival follow-up of 12.8 months). These data suggest that MRP4 expression may influence PDAC patient outcome, and that high MRP4 levels could be indicators of poor survival.

MRP4 Expression in PDAC Cell Lines Is Associated with cAMP Extrusion. To further understand the role of MRP4 in PDAC, we evaluated MRP4 expression levels and functionality in three human PDAC cell lines with different degrees of differentiation and, therefore, malignancy: HPAF-II (well-differentiated), BxPC-3 (moderately-differentiated), and PANC-1 (poorly-differentiated) cell lines (Iwamura and Hollingsworth, 2002). Both qPCR and WB studies confirmed the endogenous expression of MRP4 in the tested cell lines (Fig. 2A) and showed an association between higher MRP4 expression levels and more undifferentiated and malignant phenotypes. These findings are consistent with previous histologic observations of MRP4 expression in human PDAC samples (Zhang et al., 2012).

Because MRP4 has been described as the main transporter involved in cAMP extrusion in several cell types (Van Aubel et al., 2005; Copsel et al., 2011), we evaluated the association between MRP4 expression levels and this process in PDAC cells. Treatment with $25 \mu \mathrm{M}$ forskolin (an adenylyl cyclase direct activator) induced a rapid increase in i-cAMP levels during the first 15 minutes of incubation in all cell lines, followed by a delayed increment in e-cAMP levels (Fig. 2B). We also characterized the cAMP exclusion process in the presence of a physiologic stimulus by treating the three cell lines with the $\mathrm{G} \alpha$ s protein-coupled receptor agonists, amthamine $(10 \mu \mathrm{M})$ and isoproterenol $(10 \mu \mathrm{M})$, targeting the histaminereceptor 2 and the $\beta$-adrenergic receptor, respectively. As expected, both ligands produced an increment in i-cAMP levels with a concomitant rise in e-cAMP concentrations $(P<$ 0.05, Supplemental Fig. 1). Given that the maximum i-cAMP levels (values per $10^{5}$ cells: HPAF-II $124 \pm 16$ pmol; BxPC-3 $65.4 \pm 7.4$ pmol; and PANC-1 $43.0 \pm 3.8$ pmol) reached upon forskolin stimulation varied significantly $(P<0.05)$ between the three cell lines tested, we plotted area under the curve (AUC) values for e-cAMP/AUC of i-cAMP levels from the data in Fig. 2B, which were obtained from kinetic assays with forskolin ( $25 \mu \mathrm{M}, 60$ minutes). This ratio is an indicator of cAMP efflux activity. Figure 2C shows that PANC-1 cells present the highest $\mathrm{AUC}_{\text {e-cAMP}} / \mathrm{AUC}_{\mathrm{i}-\mathrm{AMP}}$, followed by
BxPC-3 cells and HPAF-II cells, which portray the lowest value $(P<0.01)$. To confirm these results, we performed forskolin concentration-response curves to compare the response of each cell line to different i-cAMP levels as indicative of their cAMP efflux activity (Fig. 2D). PANC-1 cells depict the greatest capacity to exclude cAMP to the extracellular compartment, because they present higher e-cAMP levels at lower concentrations of i-cAMP, whereas BxPC-3 cells show intermediate capacity, and HPAF-II cells portray the lowest exclusion capacity for this substrate (Fig. 2D). Furthermore, when comparing the three cell lines, higher MRP4 expression levels (Fig. 2A, $P<0.05$ ) were accompanied by higher efflux activity (Fig. $2 \mathrm{C}, P<0.01$ ) and vice versa, thus indicating an association between both aspects.

To confirm MRP4-specific involvement in cAMP extrusion, we performed studies using MRP4-specific silencing or overexpression in PDAC cell lines. We established both MRP4-silenced PANC-1 (Fig. 3A, $P<0.001$ ) and a MRP4overexpressing BxPC-3 clone (Fig. 3B, $P<0.01$ ) and evaluated cAMP response upon forskolin $(25 \mu \mathrm{M}$, maximum 60 minutes) stimulation. Specific silencing in PANC-1 cells using two nonoverlapping shRNA sequences (MRP4sh1 and MRP4sh2 cells) led to a decrease in cAMP extrusion upon forskolin stimulation $(P<0.001)$ with no significant changes in i-cAMP levels when compared with control cells (Fig. 3C). Conversely, the stable MRP4-overexpressing BxPC-3 clone (BxPC-3 MRP4) led to an increment in cAMP extrusion upon forskolin stimulation $(P<0.001)$ and showed significant lower levels of i-cAMP compared with the control clone ( $P<0.001$, BxPC-3 mock, Fig. 3D). Moreover, basal cAMP extrusion was diminished in PANC$1 \mathrm{MRP} 4 \mathrm{sh} 1(P<0.05)$ and augmented in the BxPC-3 MRP4 clone $(P<0.01)$ compared with their controls over a period of 1-6 hours without forskolin stimulation, indicating that MRP4 is the main participant in the cAMP exclusion process in these cell lines (Fig. 3E). No significant differences were observed in i-cAMP basal levels between PANC1 MRP4sh1 and PANC-1 scramble cells, or between BxPC-3 MRP4 and BxPC-3 mock cells.

The next step was to evaluate the ability of different wellknown MRP4 inhibitors to modulate i-cAMP and e-cAMP levels after forskolin stimulation. To that end, we performed concentration-response curves after preincubation (3 minutes) with different concentrations of probenecid (a nonspecific anion transporter inhibitor), followed by treatment with forskolin (33 $\mu \mathrm{M}, 30$ minutes) in the three PDAC cell lines (Fig. 4A). Preincubation with probenecid produced a concentration-dependent decrease in the e-cAMP levels achieved by forskolin treatment, and concentrationresponse curves adjusted a similar apparent $\mathrm{IC}_{50}$ for the 


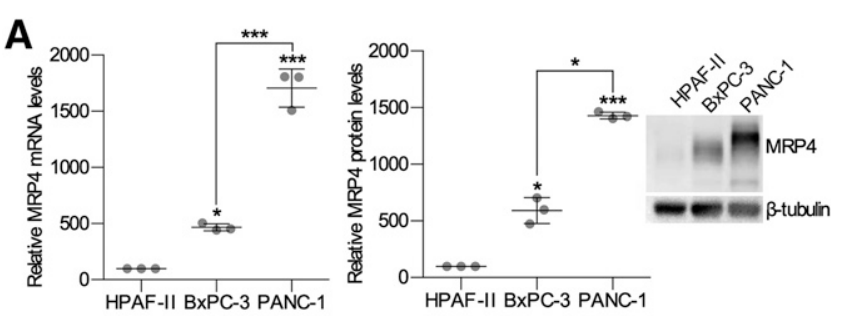

B
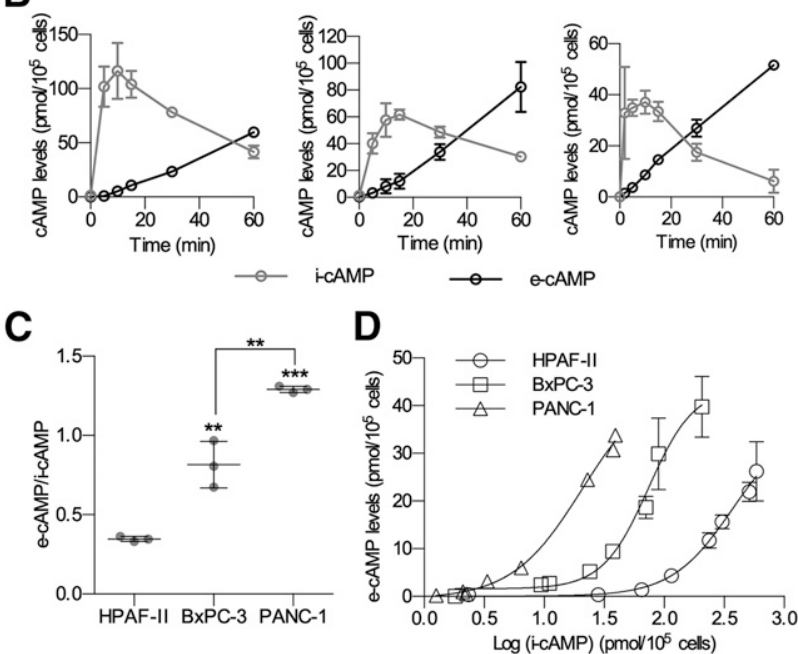

Fig. 2. MRP4 expression and cAMP extrusion capacity of PDAC cell lines. (A) Relative MRP4 mRNA (left) and protein (middle and right) basal expression levels in PDAC cell lines as determined by qPCR and WB, respectively. WB results were normalized to $\beta$-tubulin as a loading control, and $\mathrm{qPCR}$ results were normalized to $\beta$-actin as a housekeeping gene; both were expressed relative to $\mathrm{HPAF}$-II MRP4 levels. A representative WB assay of three independent experiments is shown (right). Statistics: oneway analysis of variance, ${ }^{*} P<0.05 ; * * P<0.01 ; * * * P<0.001$ vs. HPAF-II or between data when indicated. (B) Kinetic studies of cAMP production and extrusion processes. i-cAMP and e-cAMP levels measured by RBP in PDAC cell lines after treatment with $25 \mu \mathrm{M}$ forskolin at different time lapses (0-60 minutes). (C) The ratio of the AUC values for total i-cAMP and e-cAMP levels from kinetic experiments with forskolin $(25 \mu \mathrm{M}$, 60 minutes) was plotted for each of the PDAC cell lines. Data are shown as mean \pm S.D. $(n=3)$. Statistics: one-way analysis of variance. $* * P<$ $0.01 ; * * * P<0.001$ vs. HPAF-II or between data when indicated. (D) cAMP extrusion capacity of PDAC cell lines. i-cAMP and e-cAMP levels were measured by RBP after 30-minute treatment with different forskolin concentrations $(0-333 \mu \mathrm{M})$. Results are shown as e-cAMP vs. i-cAMP levels. (A-D) Data are shown as mean \pm S.D. $(n=3)$.

three cell lines (Fig. 4A). Conversely, i-cAMP levels were not significantly modified with none of the tested probenecid concentrations. We also evaluated the effect of MK571 (a MRP1, 2, and 4 inhibitor) on i-cAMP and e-cAMP levels in response to forskolin stimuli (Fig. 4B). In the three cell lines, preincubation with MK571 (25 $\mu \mathrm{M}, 5$ minutes) led to a great decrease in e-cAMP levels $(P<0.001)$, although no significant differences in i-cAMP levels could be observed 30 minutes after stimuli (Fig. 4B). To evaluate cAMP levels upon stimulation at a shorter time scale, we performed kinetic studies in PANC-1 cells after stimulation with forskolin $(25 \mu \mathrm{M})$, with or without preincubation with MK571 $(25 \mu \mathrm{M})$ and measured i- and e-cAMP at different time points $(0$ 5 minutes) by RBP assay (Fig. 4C). Lower e-cAMP levels were observed in PANC-1 cells pretreated with MK571 starting 3 minutes after forskolin stimulation compared with control cells $(P<0.01)$. Also, MK571-treated cells presented higher i-cAMP levels 5 minutes after forskolin stimulation $(P<0.05)$. In a similar approach, we also assessed i-cAMP levels in PANC-1 cells by FRET assays using Epac-S ${ }^{\mathrm{H} 187}$ as a cAMP molecular sensor in the presence of isoproterenol $(0.5 \mu \mathrm{M})$ stimulation, with or without preincubation with MK571 (25 $\mu \mathrm{M}, 3$ minutes). Higher AUC values ( $0-5$ minutes, $P<0.05)$, together with a delay in the maximum cAMP response to isoproterenol, were observed when MRP4 was inhibited with MK571 (Fig. 4D). Given that we observed that the MRP4 inhibitors modified both i- and e-cAMP levels in short time lapses in the presence of stimulus (forskolin or isoproterenol), we set out to evaluate their response in basal conditions. To do so, we measured i-cAMP levels in BxPC-3 cells by FRET assays using the same molecular sensor after treatment with probenecid, MK571, or ceefourin-1 (a novel MRP4-specific inhibitor) (Cheung et al., 2014) (Fig. 4E). Figure 4E shows 7 minutes i-cAMP kinetics after the addition of $100 \mu \mathrm{M}$ corresponding inhibitor. To compare potency and efficacy to raise i-cAMP levels, we performed concentration-response experiments. Probenecid proved to be the most potent and effective inhibitor $\left[\mathrm{pEC}_{50}(\mathrm{M})=3.75 \pm 0.08\right]$, followed by MK571 $\left[\mathrm{pEC}_{50}\right.$ $(\mathrm{M})=3.23 \pm 0.10$ ] and ceefourin-1, for which potency could not be calculated properly due to deficiently fit value estimations $\left[\mathrm{pEC}_{50}\right.$ (M) $<3.83$ ]

Finally, to test the long-term capacity of the inhibitors to alter e-cAMP in absence of stimuli, we used PANC-1 cells, which showed the greatest capacity to exclude cAMP (Fig. 4F). As expected, we observed a significant reduction in e-cAMP levels produced by treatment with the three inhibitors in basal conditions $(P<0.05)$. Collectively, these results confirm that MRP4 inhibition affects temporal i-cAMP and e-cAMP levels upon stimulation and in basal conditions in PDAC cell lines.

The Blockage of MRP4 Activity Inhibits PDAC Cell Proliferation through the Activation of i-cAMP/Epac/Rap1 Signaling Pathway and the Modulation of e-cAMP Levels. To assess the influence of MRP4 on PDAC cell line growth, we first evaluated the biologic effect of its pharmacological inhibition. Treatment with probenecid $(0.5 \mathrm{mM})$, as well as with MK571 (25 $\mu \mathrm{M})$, inhibited cell proliferation in the three cell lines $(P<0.01$, Fig. 5A). It is worth noting that none of the tested concentrations decreased cell viability, thus discarding any cytotoxic effects that might account for this observation (data not shown). We further analyzed cell cycle distribution in PANC-1 cells after MRP4 pharmacological inhibition. An increase in G0/G1 subpopulation was observed upon probenecid $(P<0.001)$ or MK571 $(P<0.05)$ treatment, with a concomitant reduction in the percentage of cells in $\mathrm{S}$ phase (Fig. 5B).

To ensure specificity of the antiproliferative response of MRP4 inhibition, we performed kinetic cell proliferation assays and cell cycle analysis using the silenced PANC-1 MRP4sh1 clone versus PANC-1 scramble cells and BxPC-3 MRP4 clone versus BxPC-3 mock cells. Interestingly, silenced PANC-1 cells exhibited a slower doubling time $(\mathrm{Td}=41.0 \pm 7.4$ hours $)$ than PANC-1 scramble cells ( $\mathrm{Td}=33.5 \pm 2.9$ hours) used as control ( $t$ test, $P<0.05$, Fig. 5C). In agreement with previous findings (Zhang et al., 2012), cell cycle analysis highlighted that silencing MRP4 increased the G0/G1 population $(P<0.001)$ with a concomitant reduction of cells in $\mathrm{S}$ phase $(P<0.05)$ compared with PANC-1 scramble cells (Fig. 5D). Moreover, BxPC-3 MRP4 cells portrayed a faster doubling time $(\mathrm{Td}=12.7$ \pm 2.0 hours) compared with the mock cells $(\mathrm{Td}=22.1 \pm 6.7$ hours) used as control ( $t$ test, $P<0.05$, Fig. 5E). Unsurprisingly, when cell cycle was analyzed, an increment in the $\mathrm{S}(P<0.01)$ 


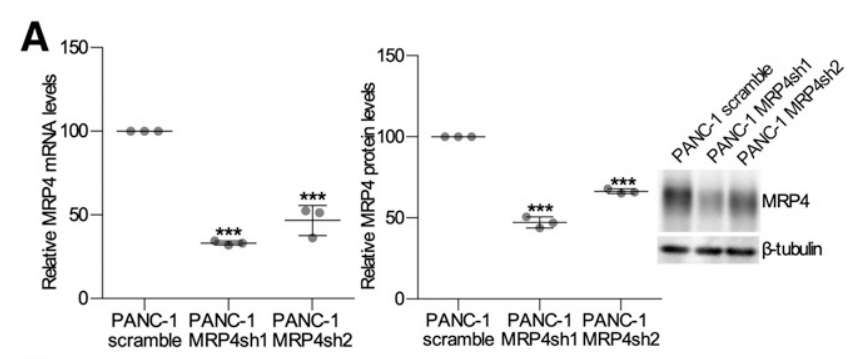

B
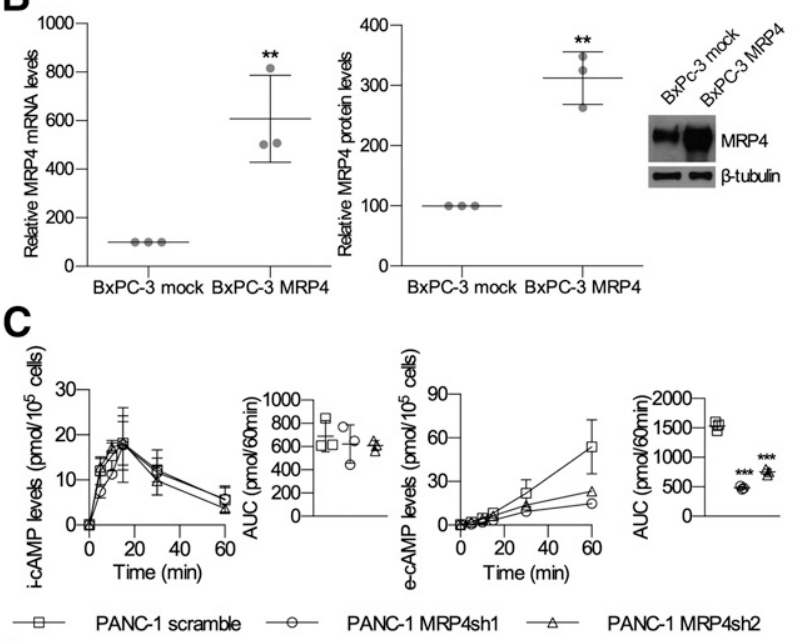

D
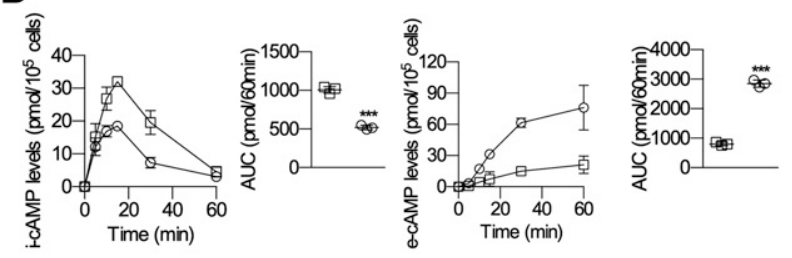

$\square \quad B \times P C-3$ mock
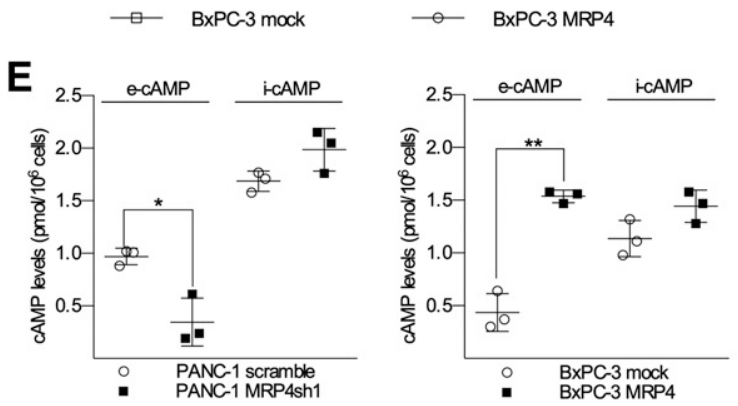

Fig. 3. MRP4 silencing or overexpression in PDAC cell lines alters the cAMP extrusion capacity. (A) Relative MRP4 mRNA (left) and protein (middle and right) basal expression in PANC-1 MRP4-silenced clones determined by qPCR and WB, respectively. WB results were normalized to $\beta$-tubulin, and qPCR results were normalized to $\beta$-actin and both were expressed relative to PANC-1 scramble MRP4 levels. A representative WB assay of three independent experiments is shown (right). Statistics: oneway analysis of variance vs. PANC-1 scramble. (B) Relative MRP4 mRNA (left) and protein (middle and right) basal expression in BxPC-3 MRP4overexpressing clone determined by $\mathrm{qPCR}$ and $\mathrm{WB}$, respectively. WB results were normalized to $\beta$-tubulin as a loading control, and qPCR results were normalized to $\beta$-actin as a housekeeping gene; both were expressed relative to $\mathrm{BxPC}-3$ mock MRP4 levels. A representative WB assay of three independent experiments is shown (right). Statistics: $t$ test. (C and D) Kinetic studies of cAMP production and extrusion processes after MRP4 silencing and overexpression. i-cAMP (left) and e-cAMP (right) levels measured by RBP in PANC-1 MRP4-silenced clones and BxPC-3 MRP4-overexpressing clones after treatment with $25 \mu \mathrm{M}$ forskolin for different time lapses (0-60 minutes). AUC values for total i-cAMP and e-cAMP levels of each condition tested are also shown as a bar plot (right of curve plot). Statistics: (C) one-way analysis of variance, vs. PANC-1 and G2/M $(P<0.01)$ subpopulations was observed in BxPC-3 MRP4 compared with BxPC-3 mock cells (Fig. 5F).

Once proven that MRP4 inhibition or silencing impairs cell proliferation and cell cycle distribution in PDAC cell lines, we selected probenecid for further experiments because it showed to be the most potent and effective inhibitor among those tested (Fig. 4E) and it has the advantage of being Food and Drug Administration (FDA) approved, which makes it a good candidate for drug repositioning (Ahmed et al., 2016).

It has been previously reported that MRP4 silencing reduces proliferation of PDAC cell lines, but the underlying mechanism has not yet been described (Zhang et al., 2012). Because we observed that MRP4 pharmacological inhibition produced a prompt increment of i-cAMP levels with a concomitant decrease in e-cAMP levels and that this phenomenon is sustained in time, we evaluated both e- and i-cAMP levels and how this affected cell proliferation. We first studied whether the addition of e-cAMP $(10 \mu \mathrm{M})$ was able to reverse the antiproliferative effect of MRP4 inhibition. Remarkably, treatment with cAMP partially reversed the antiproliferative effect of MRP4 silencing and pharmacological inhibition in PDAC cell lines $(P<0.05)$, although it did not alter their basal growth rate (Fig. 6A). Accordingly, exposure to e-cAMP restored the cell cycle distribution of MRP4-silenced PANC-1 cells, significantly decreasing the G0/G1 subpopulation $(P<0.05$, Fig. 6B). Collectively, these results reveal a novel role of e-cAMP in pancreatic cancer cell proliferation. To determine whether the mitogenic effect of e-cAMP is mediated by any of its extracellular metabolites, PANC-1 cells were cotreated with probenecid $(0.5 \mathrm{mM})$ and 5 'AMP $(10 \mu \mathrm{M})$ or adenosine $(10 \mu \mathrm{M})$. Interestingly, neither metabolite could rescue cell proliferation after MRP4 pharmacological inhibition (Supplemental Fig. 2A). Similar results were observed when MRP4-silenced PANC-1 cells were treated with $5^{\prime} \mathrm{AMP}(10 \mu \mathrm{M})$ or adenosine $(10 \mu \mathrm{M})$ (Supplemental Fig. 2B). In accordance, inhibition of e-cAMP degradation by 1,3-dipropyl-8-p-sulfophenylxanthine ( $5^{\prime}$ nucleotidase inhibitor, $10 \mu \mathrm{M}$ ) or Adenosine 5'-[alpha,betamethylene]diphosphate (AMPCP, ecto-PDE inhibitor, $10 \mu \mathrm{M}$ ) did not abrogate its pro-proliferative effect on PANC-1 scramble and MRP4-silenced PANC-1 cells $(P<0.05$, Supplemental Fig. 2C). Altogether, these results demonstrate that e-cAMP can act per se as an autocrine/paracrine growth factor in PDAC cells and indicate that the MRP4-mediated cAMP extrusion process provides a mitogenic signal from the extracellular compartment.

We next studied which of the i-cAMP effectors are involved in the antiproliferative response triggered by MRP4 inhibition. Experiments in human coronary artery endothelial cells have shown that MRP4-specific silencing decreases cell proliferation by activating the cAMP/PKA/cAMP response element binding protein pathway (Sassi et al., 2008). However, in PANC-1 cells, the pharmacological inhibition of PKA with

scramble. (D) $t$ test. (E) Basal cAMP levels after MRP4-specific silencing (left) or oveexpression (right). i-cAMP and e-cAMP levels measured by RBP in PANC-1 scramble and PANC-1 MRP4sh1 cells after 60-minute incubation in fresh culture media and in BxPC-3 mock and BxPC-3 MRP4 after 360-minute incubation in fresh culture media. Statistics: $t$ test. (A-E) Data are shown as mean \pm S.D. $(n=3)$. $* P<0.05 ; * * P<0.01 ; * * * P<$ 0.001 . 

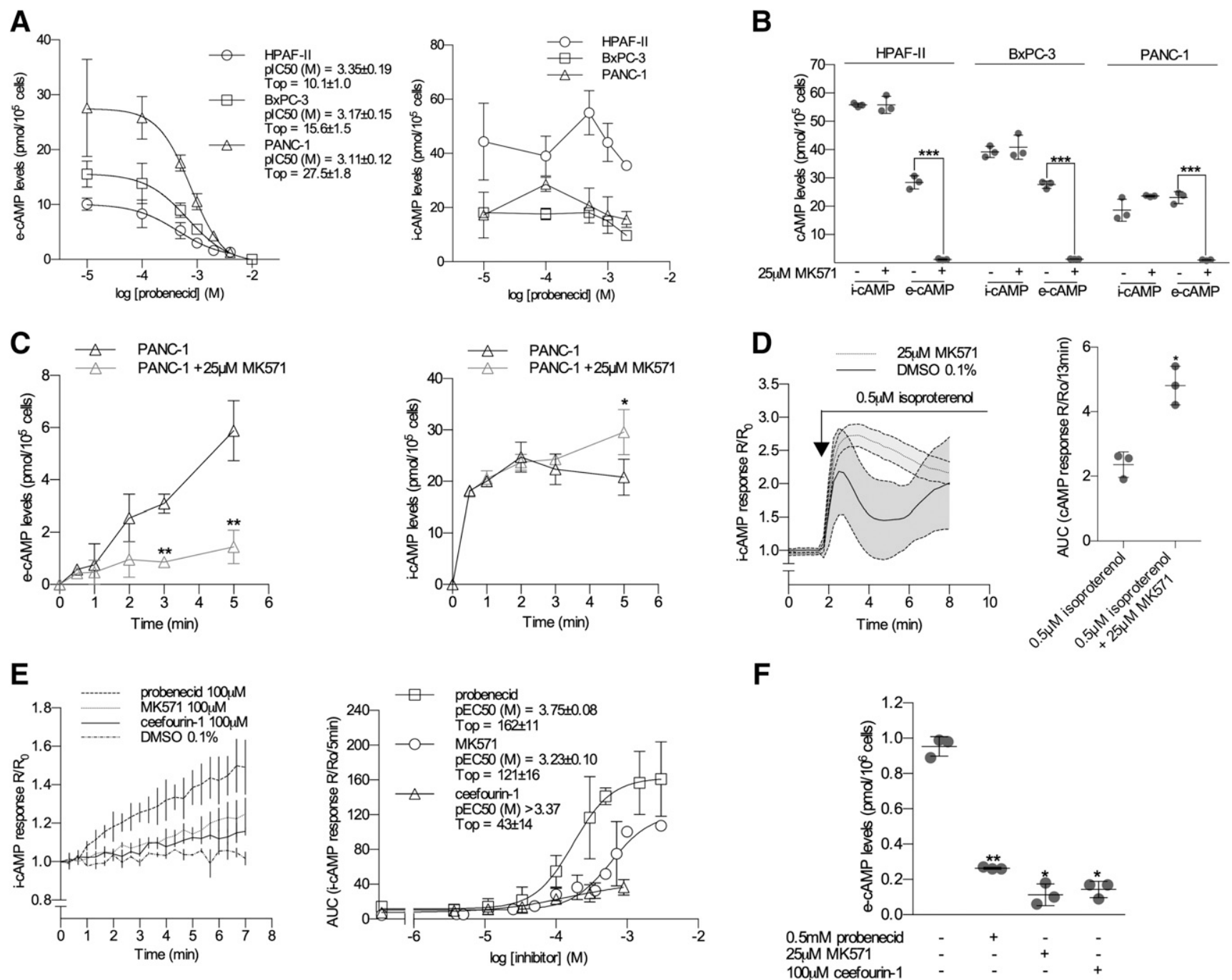

Fig. 4. MRP4 inhibitors' effect on cAMP extrusion in PDAC cell lines. (A) Effect of probenecid (0.00001-0.002 M) on i-cAMP and e-cAMP levels in human PDAC cell lines. i-cAMP and e-cAMP levels were measured by RBP after 3-minute preincubation with probenecid at the indicated concentrations, followed by treatment with $33 \mu \mathrm{M}$ forskolin for 30 minutes. e-cAMP vs. log of probenecid concentration assays were fit to a four-parameter concentrationresponse curve. Pool data of all experiments were fit to a single curve. Statistics: one-way analysis of variance. Data are shown as mean \pm S.D. $(n=4)$. Statistics: one-way analysis of variance. (B) Endpoint studies of cAMP production and extrusion processes after MRP4 inhibition with MK571. i-cAMP and e-cAMP levels were measured by RBP after 30-minute treatment with $25 \mu \mathrm{M}$ forskolin in PDAC cell lines preincubated for 5 minutes with $25 \mu \mathrm{M}$ MK571. Data are shown as mean \pm S.D. $(n=3)$. Statistics: $t$ test. (C) Kinetic studies of cAMP production and extrusion processes after MRP4 inhibition with MK571. i-cAMP (right) and e-cAMP (left) levels measured by RBP in PANC-1 cells preincubated for 5 minutes with $25 \mu \mathrm{M}$ MK571 after treatment with $25 \mu \mathrm{M}$ forskolin for different time lapses (0-5 minutes). Statistics: $t$ test. Data are shown as mean \pm S.D. $(n=3)$. (D) Real-time temporal progression of i-cAMP levels. Time course of FRET changes measured in single PANC-1 cells transiently transfected with the Epac-S ${ }^{\text {H187 }}$ cAMP biosensor. Cells were pretreated for 5 minutes with either $25 \mu \mathrm{M}$ MK571 or $0.1 \%$ DMSO (vehicle control), and, after this treatment, cells were stimulated with $0.5 \mu \mathrm{M}$ isoproterenol. Maximum cAMP response was observed 45 seconds after adding isoproterenol and 1.5 minutes in the presence of MK571. Traces are representative of four independent experiments (mean \pm S.D., six to eight cells per treatment, $n=3$ ). AUC values for total cAMP response for each tested condition are also shown as a scatter plot (right of the curve plot). Statistics: $t$ test. (E) Effect of pharmacological MRP4 inhibition on i-cAMP levels. Time course of FRET changes in stable BxPC-3 Epac-S ${ }^{\mathrm{H} 187}$ cells measured in a Flex Station 3 after the addition of the corresponding inhibitors (100 $\mu \mathrm{M}$, 7 minutes). Traces are representative of three or four independent experiments (mean \pm S.D.). Concentration-response curves for the three inhibitors were constructed integrating the AUC of 5-minute $R / R_{0}$ i-cAMP response for each concentration of all experiments. Pool of AUC values of 5-minute $R / R_{0}$ i-cAMP response vs. log of concentration of each inhibitor was fit to a four-parameter concentration-response curve. $\mathrm{EC}_{50}$ and top values are reported for each inhibitor. (F) Basal e-cAMP levels in PANC-1 cells after MRP4 pharmacological inhibition. e-cAMP levels were measured by RBP in PANC-1 cells treated with $0.5 \mathrm{mM}$ probenecid, $25 \mu \mathrm{M}$ MK571, or $100 \mu \mathrm{M}$ ceefourin-1 after 60-minute incubation in fresh culture media. Statistics: one-way analysis of variance vs. control. Data are shown as mean \pm S.D. $(n=3)$. (A-F) $* P<0.05 ; * * P<0.01$; *** $P<0.001$.

KT5720 $(1 \mu \mathrm{M})$, or its inactivation by expressing a PKA inhibitor peptide (PKI), did not revert the antiproliferative effect of MRP4 silencing (Fig. 6C), suggesting the involvement of another cAMP effector. In addition to PKA, i-cAMP can activate the Rap1-specific exchange factor Epac. In fact, it has been proposed that the activation of the Epac/Rap1 cascade could mediate cAMP-dependent growth inhibition in PDAC cells (Lorenz et al., 2008). Therefore, we next evaluated the role of Epac and Rap1 on the antiproliferative effect of MRP4 inhibition. To attenuate this signaling pathway, we transiently transfected PANC-1 cells either with the Epac dominant-negative mutant (N-Epac) or with the Rap1-GAP construct that accelerates Rap1-GTP hydrolysis favoring its inactive form. Both strategies abolished the effects of MRP4 pharmacological inhibition $(P<0.05)$ or silencing $(P<0.05)$ on cell proliferation, indicating that MRP4 modulates 


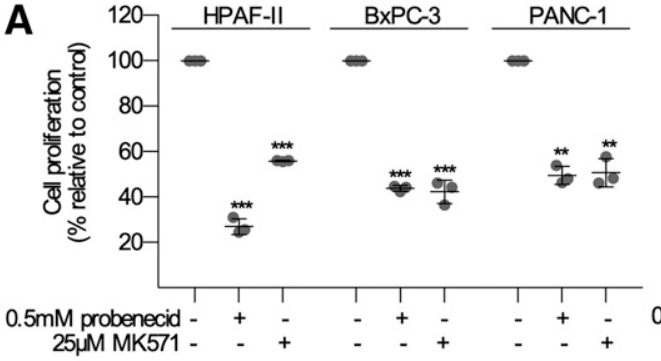

C

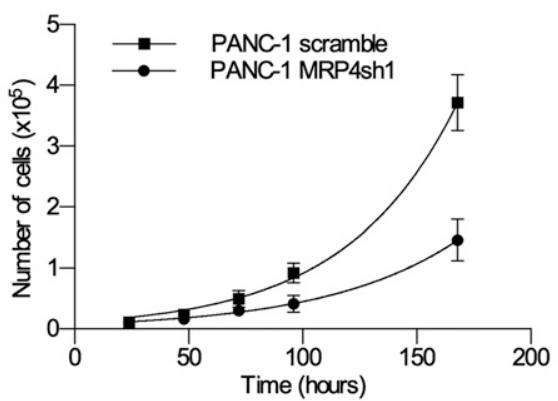

E

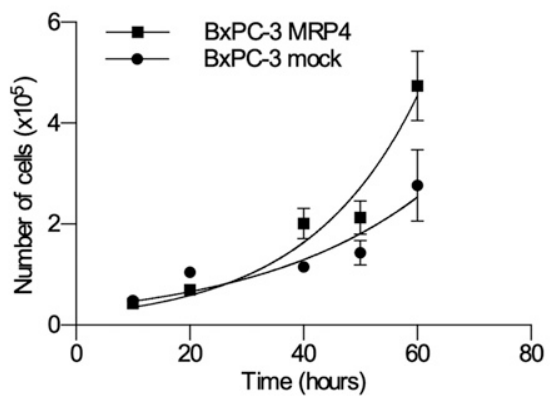

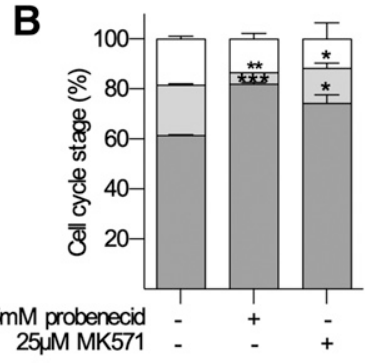
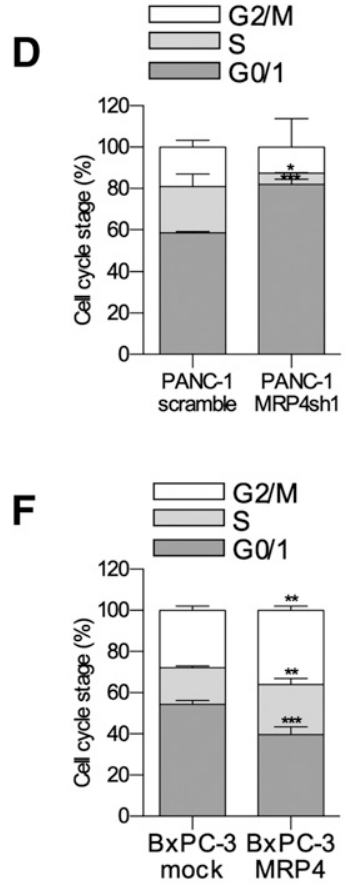

Fig. 5. Role of MRP4 in cell proliferation. (A) Cell proliferation in response to MRP4 inhibition. Cell proliferation of PDAC cell lines in the presence of $0.5 \mathrm{mM}$ probenecid or $25 \mu$ M MK571 determined by cell count with a hemocytometer chamber after 72-hour incubation in fresh culture media. Results are expressed relative to the corresponding control group. Statistics: one-way analysis of variance vs. control. (B) Cell cycle analysis after MRP4 pharmacological inhibition. Cell cycle phase distribution of synchronized PANC-1 cells, treated with $0.5 \mathrm{mM}$ probenecid or $25 \mu \mathrm{M}$ MK571 for 72 hours in fresh culture media, was determined by flow cytometry. Statistics: one-way analysis of variance vs. control. (C) Cell proliferation of PANC-1 MRP4sh1 and PANC-1 scramble cells was determined by total cell counting with a hemocytometer chamber. (D) Cell cycle phase distribution of PANC-1 MRP4sh1 and PANC-1 scramble cells was determined by flow cytometry in exponentially growing cells after 72-hour incubation in fresh culture media. Statistics: $t$ test. (E) Cell proliferation of BxPC-3 MRP4 and BxPC-3 mock was determined by total cell counting with a hemocytometer chamber. (F) Cell cycle phase distribution of BxPC-3 MRP4 and BxPC-3 mock cells was determined by flow cytometry in exponentially growing cells after 72-hour incubation in fresh culture media. Statistics: $t$ test. (A-F) $* P<0.05 ; * * P<0.01$; *** $P<0.001$ vs. control. Data are shown as mean \pm S.D. $(n=3)$. (C and E) Data were adjusted to an exponential growth equation using Prism 6.0 (GraphPad Software).
PANC-1 cell growth through the cAMP/Epac/Rap1 pathway (Fig. 6D)

In conclusion, our findings show that the antiproliferative effect of MRP4 inhibition in PDAC cells is a consequence of cAMP augmentation in the intracellular compartment and a deficiency in the extracellular compartment. The overall response is the activation of the Epac/Rap1 pathway that abrogates cell proliferation.

\section{Discussion}

The clinical outcome for PDAC patients remains dismal, and it is clear that there is an urgent need to understand more about the molecular mechanisms of PDAC pathogenesis and progression to develop an effective treatment.

Among other participants involved in cancer development, the role of cAMP signaling pathway in this disease has been long known (Fajardo et al., 2014); every important component of its pathway has been taken into consideration as a potential cancer drug target (Naviglio et al., 2009; Dou and Wang, 2010; Savai et al., 2010). However, although effective, these strategies exhibit toxicity in normal tissues, probably due to the ubiquitous expression of their molecular targets (Propper et al., 1999; Schwede et al., 2000). One of the key participants in the regulation of the cAMP pathway is MRP4, due to its ability to extrude cyclic nucleotides to the extracellular space.

Regarding pancreatic cancer, a recent study involving human pancreatic samples reports that the expression level of MRP4 is consistently augmented compared with paratumor tissues, which supports the notion that MRP4 may promote carcinogenesis (Zhang et al., 2012). Our present study shows that patients with higher levels of MRP4 expression tend to have a shorter overall survival than those with lower levels of MRP4. Thus, a high level of MRP4 expression in PDAC patients seems to predict a poor prognosis.

In agreement with previous observations (Zhang et al., 2012), our data show that high MRP4 expression is associated with low pancreatic cancer cell line differentiation stages. Likewise, it is well established that cAMP is involved in the differentiation of many types of cancer cells (Prasad and Kumar, 1975; Klein and Loizzi, 1977; Prasad et al., 2003; Moreno et al., 2006; Copsel et al., 2011). In accordance, MRP4 is upregulated in cancer stemlike cells (CSC) (Oevermann et al., 2009; Warrier et al., 2012), and its inhibition results in CSC differentiation (Copsel et al., 2014), which may suggest that undifferentiated cells require active extrusion of cAMP, or other MRP4 endogenous substrate, to maintain their pluripotent or immature state.

Unlike other components of the cAMP pathway that are vastly expressed and widely distributed in the body, MRP4 
A
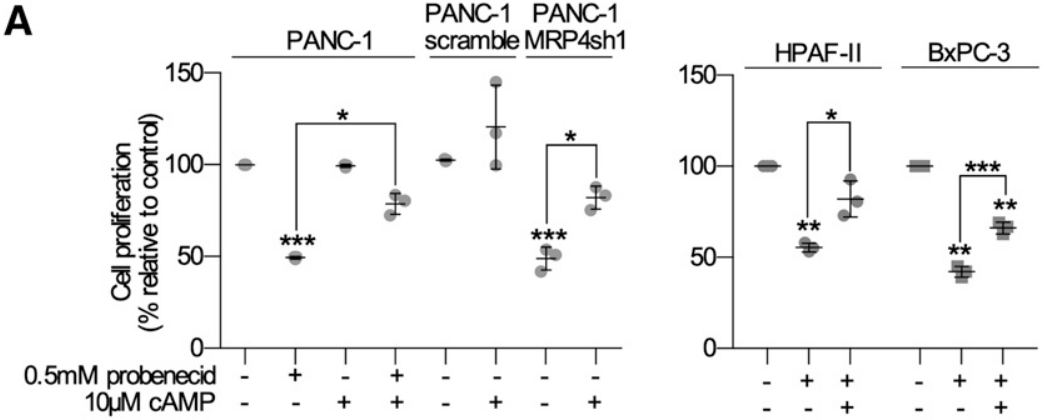

B

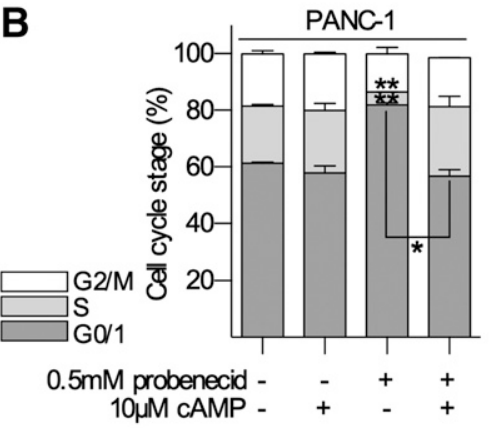

C

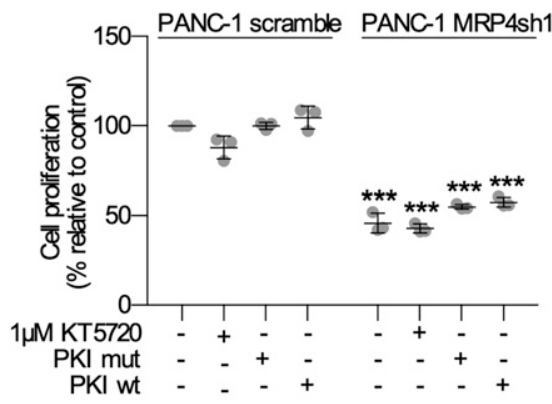

D

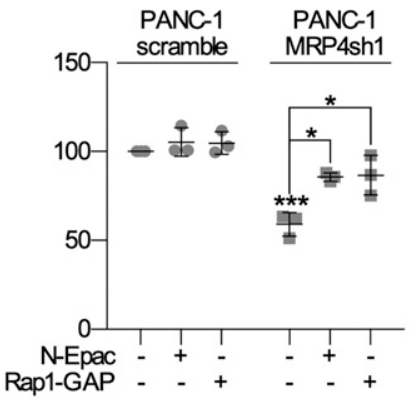

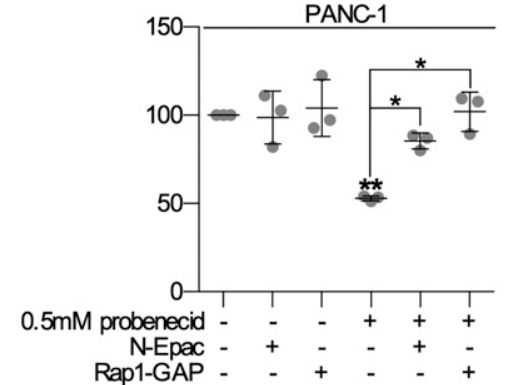

Fig. 6. Pharmacological inhibition or silencing of MRP4 inhibits PDAC cell proliferation by activating the cAMP/ Epac/Rap1 signaling pathway. (A) Reversion of the antiproliferative effect of MRP4 inhibition and specific silencing mediated by e-cAMP. Cell proliferation of synchronized PANC-1, HPAF-II, and BxPC-3 cells preincubated with $0.5 \mathrm{mM}$ probenecid, or PANC-1 scramble and PANC-1 MRP4sh1 cells, was determined by cell count in a hemocytometer chamber after 72-hour treatment with $10 \mu \mathrm{M}$ cAMP. Statistics: two-way analysis of variance (to compare between different cell lines) and $t$ test (to compare treatments from the same cell line); right panel, one-way analysis of variance vs. control or between data, as indicated. (B) Effect of e-cAMP addition on cell cycle after MRP4 pharmacological inhibition. Cell cycle phase distribution of synchronized PANC-1 cells pretreated with $0.5 \mathrm{mM}$ probenecid was determined by flow cytometry after 72 hours of incubation with $10 \mu \mathrm{M}$ cAMP. Statistics: one-way analysis of variance vs. control or between bars, as indicated. (C) PKA pathway inhibition effects on cell proliferation in MRP4-silenced PANC-1 cells. Cell proliferation of PANC-1 scramble and PANC-1 MRP4sh1 cells, either transiently transfected with pGFP-PKI (codes for a wild-type PKA inhibitor peptide) and pGFP-PKImut (codes for an inactive PKA inhibitor peptide) constructs or treated with 1 KT5720 (PKA pharmacological inhibitor), was determined by cell count in a hemocytometer chamber after 72 hours of incubation. Statistics: two-way analysis of variance. (D) Reversion of the antiproliferative effect of MRP4 inhibition mediated by Epac/Rap1 inhibition. PANC-1 cell proliferation in response to $0.5 \mathrm{mM}$ probenecid, and PANC-1 scramble and PANC-1 MRP4sh1 cells transiently transfected with either a negative-dominant mutant of Epac (N-Epac) or a construction that increases Rap1 inactive form (Rap1-GAP), was monitored by cell count in a hemocytometer chamber 72 hours after incubation. Statistics: two-way analysis of variance (between different cell lines) and one-way analysis of variance (within same cell lines); right panel, one-way analysis of variance vs. control or between data, as indicated. (A-D) $* P<0.05$; ** $P<0.01$; $* * * P<0.001$ vs. control or between data when indicated. Data are shown as mean \pm S.D. $(n=3)$. is highly expressed in kidneys and prostate, whereas lower expression levels have been reported in other organs (Borst et al., 2007; Wen et al., 2015). Some of the physiologic functions of this transporter were identified in experiments carried out in $\mathrm{Mrp}^{-/-}$mice models and include the activation of platelets and formation of thrombus (Decouture et al., 2015), testosterone testicular production (Morgan et al., 2012), and regulation of prostaglandin E metabolite plasma levels that control the inflammatory pain threshold (Lin et al., 2008). Nevertheless, these mice did not show major physiologic alterations (Russel et al., 2008), indicating that MRP4 inhibition could be a feasible therapeutic approach. Also, exposition of the knockout mice to several drugs highlighted MRP4 role in the excretion of such agents, as they presented altered cytotoxicity and tissue distribution compared with wild-type animals (Leggas et al., 2004; Zamek-Gliszczynski et al., 2006; Takenaka et al., 2007; Liu et al., 2017). Moreover, several FDA-approved drugs inhibit MRP4 activity without showing any serious adverse effects (Yaneff et al., 2017). However, to date, no pharmacological selective inhibitors of MRP4, with proven in vivo efficacy, are available. The most widely used MRP4 inhibitors, such as probenecid and MK571, also have inhibitory activity against other transporters (Keppler, 2011) and PDE (Xie et al., 2011). Recently, two selective MRP4 inhibitors have been described
(Cheung et al., 2014), but have not been fully characterized yet, and their suitability for in vivo studies is still unknown. In this work, we prove the efficacy of probenecid, MK571, and ceefourin-1 to inhibit MRP4 activity and, consequently, enhance i-cAMP and diminish e-cAMP levels.

The FDA has approved the use of probenecid for the treatment of gout and hyperuricemia four decades ago. This drug increases excretion of uric acid through urine and, if used in the recommended doses, has only a few and nonserious side effects (Ahmed et al., 2016). Notably, oral administration of probenecid in the average clinically used dose (2 g/day) in healthy male volunteers causes a mean plasmatic concentration of $148.6 \mu \mathrm{g} / \mathrm{ml}(520.67 \mu \mathrm{M})$ (Selen et al., 1982), which is enough to inhibit PDAC cell proliferation according to our in vitro studies. Altogether, this points out the potential clinical relevance of its implementation in the treatment of PDAC in combination with cytotoxic agents (Fury et al., 2006). Additionally, because probenecid blocks several MRPs simultaneously, its effect could enhance chemotherapeutic drugs, such as 5-fluorouracil and gemcitabine, which are used to treat PDAC (Conroy et al., 2011; Von Hoff et al., 2013; Middleton et al., 2014; Adamska and Falasca, 2018). However, more studies in this matter, such as preclinical in vivo assays, should be performed to test the efficacy of the proposed combined therapy together with the possibility of consequent 
drug-drug interactions. Besides cAMP, MRP4 has the ability to extrude a wide variety of exogenous and endogenous substances (Russel et al., 2008; Yaneff et al., 2017). One of the major problems in PDAC therapy is multidrug resistance, which not only leads to a reduced efficacy of chemotherapeutic drugs, but can also favor the survival and expansion of resistant cancer cells, thus contributing to relapses that worsen outcomes over time (Choi, 2005; Long et al., 2011; Murray et al., 2017). Interestingly, it has been described that drug-resistant cells and CSC from relapsed tumors are enriched in MRP4, even if the clinically used drugs are not MRP4 substrates (Tian et al., 2006; Hagmann et al., 2009; Adema et al., 2014; Moitra, 2015; Adamska and Falasca, 2018). These observations indicate MRP4 inhibition could be a promising strategy for PDAC treatment, allowing potential synergetic combinations with currently used chemotherapeutic drugs by both potentially enhancing their effectiveness and/or by targeting a resistant cell subpopulation. Furthermore, the fact that MRP4 can also export endogenous prostaglandin $\mathrm{E}_{2}$, a key component in inflammatory pathways, may show additional benefits for this therapeutic strategy, reducing the tumor-associated inflammation, linked to poor prognosis in pancreatic cancer (Hausmann et al., 2014).

When analyzing the role of cAMP pathway and its effectors on physiologic cellular processes such as cell proliferation, we must bear in mind that both are specific to each cell type (Stork and Schmitt, 2002; Vitali et al., 2015; Rodríguez et al., 2017). In this case, for PDAC cell lines we observed that the inhibition of MRP4 produces an indirect activation of the cAMP/EPAC/Rap1 pathway that abrogates cell proliferation. Interestingly, it has previously been reported that EPAC1 is overexpressed in pancreatic cell lines and tumor samples, and that activation of the EPAC/Rap1 cascade could mediate cAMP-dependent growth inhibition in PDAC cells (Lorenz et al., 2008). Also, consistent with our results, another study has shown that EPAC inhibition does not alter pancreatic cancer cell viability nor proliferation in basal conditions (Almahariq et al., 2013). However, when EPAC/Rap1 pathway is activated by an enhancement of $\mathrm{i}$-cAMP levels, because of MRP4 inhibition, both N-Epac and Rap1-GAP transfections that prevent activation of the pathway can restore cell proliferation. Moreover, we observed that impairment of cell proliferation caused by MRP4 inhibition was restored when cAMP was added to culture medium. Previous studies have proposed that e-cAMP can modulate a variety of cellular functions in several tissues, either by acting indirectly, through its metabolic products, or directly, through a stillunidentified receptor (Hofer and Lefkimmiatis, 2007). Further studies are needed to identify this putative cAMP receptor; thus, its existence would not only expand our understanding of the cAMP signaling pathway, but could also represent a new molecular target for PDAC treatment. Remarkably, four extracellular cAMP receptors involved in processes such as chemotaxis, cell migration, and differentiation have been described in amoeba Dictyostelium discoideum (Schaap, 2011). Recently, a cAMP ABC transporter was also described in this organism (Miranda et al., 2015). Together, these findings suggest the existence of a primitive mechanism linked to e-cAMP signaling in eukaryotic cells. Finally, the fact that MRP4 inhibition triggers a reduction in e-cAMP levels could be an invaluable tool for future clinical trials.
Almost 40 years ago, Scavennec et al. (1981) observed that cAMP concentration in urine was higher in patients with solid tumors than in healthy volunteers. Therefore, cAMP levels in urine, or even plasma, could eventually serve as an efficacy biomarker to assess the patient's response to MRP4targeted therapy.

Altogether, the present study provides direct evidence of the involvement of MRP4 in cell proliferation of PDAC cell lines through its involvement in cAMP signaling pathway. Drug repositioning of an inhibitor such as probenecid or de novo drug design of a specific inhibitor of cAMP transport by MRP4 could be appealing strategies to improve PDAC therapeutics.

\section{Acknowledgments}

pCMV-Myc-N-EPAC and pMT2-HA-Rap1GAP plasmids were provided by Dr. Omar Coso (Department of Physiology and Molecular Biology, Universidad de Buenos Aires, Argentina) (Hochbaum et al., 2003). pGFP-PKI and pGFP-PKImut plasmids were a gift of Dr. J. Silvio Gutkind (Oral and Pharyngeal Cancer Branch, National Institutes of Health) (Iglesias-Bartolome et al., 2015). The mTurquoise2-EPAC-cp173Venus-Venus (Epac-S ${ }^{\mathrm{H} 187}$ ) construct was provided by Dr. Kees Jalink (Cell Biophysics \& Imaging Group, Netherlands Cancer Institute) (Klarenbeek et al., 2015). We thank Lic. Ricardo Bello for assisting in the statistical analysis.

\section{Authorship Contributions}

Participated in research design: Carozzo, Yaneff, Gómez, Shayo, Davio.

Conducted experiments: Carozzo, Yaneff, Gómez, Di Siervi, Sahores, Diez, Attorresi, Rodríguez-González, Martin.

Contributed new reagents or analytic tools: Monczor, Fernández, Shayo, Davio.

Performed data analysis: Carozzo, Yaneff, Gómez, Di Siervi, Sahores, Diez, Martin.

Wrote or contributed to the writing of the manuscript: Carozzo, Yaneff, Gómez, Sahores, Monczor, Shayo, Davio.

\section{References}

Adamska A and Falasca M (2018) ATP-binding cassette transporters in progression and clinical outcome of pancreatic cancer: what is the way forward? World $J$ Gastroenterol 24:3222-3238.

Adema AD, Floor K, Smid K, Honeywell RJ, Scheffer GL, Jansen G, and Peters GJ (2014) Overexpression of MRP4 (ABCC4) and MRP5 (ABCC5) confer resistance to the nucleoside analogs cytarabine and troxacitabine, but not gemcitabine. Springerplus 3:732.

Ahmed MU, Bennett DJ, Hsieh T-C, Doonan BB, Ahmed S, and Wu JM (2016) Repositioning of drugs using open-access data portal DTome: a test case with probenecid (Review). Int J Mol Med 37:3-10.

Almahariq M, Tsalkova T, Mei FC, Chen H, Zhou J, Sastry SK, Schwede F, and Cheng X (2013) A novel EPAC-specific inhibitor suppresses pancreatic cancer cell migration and invasion. Mol Pharmacol 83:122-128.

Beavo JA and Brunton LL (2002) Cyclic nucleotide research -- still expanding after half a century. Nat Rev Mol Cell Biol 3:710-718.

Borst $\mathrm{P}$, de Wolf $\mathrm{C}$, and van de Wetering K (2007) Multidrug resistance-associated proteins 3, 4, and 5. Pflugers Arch 453:661-673.

Boucher MJ, Duchesne C, Lainé J, Morisset J, and Rivard N (2001) cAMP protection of pancreatic cancer cells against apoptosis induced by ERK inhibition. Biochem Biophys Res Commun 285:207-216.

Bröderdorf S, Zang S, Schaletzki Y, Grube M, Kroemer HK, and Jedlitschky G (2014) cAMP regulates expression of the cyclic nucleotide transporter MRP4 (ABCC4) through the EPAC pathway. Pharmacogenet Genomics 24: $522-526$.

Burdyga A, Conant A, Haynes L, Zhang J, Jalink K, Sutton R, Neoptolemos J, Costello E, and Tepikin A (2013) cAMP inhibits migration, ruffling and paxillin accumulation in focal adhesions of pancreatic ductal adenocarcinoma cells: effects of PKA and EPAC. Biochim Biophys Acta 1833:2664-2672.

Cancer Genome Atlas Research Network (2017) Integrated genomic characterization of pancreatic ductal adenocarcinoma. Cancer Cell 32:185-203.e13.

Carozzo A, Diez F, Gomez N, Cabrera M, Shayo C, Davio C, and Fernández N (2015) Dual role of cAMP in the transcriptional regulation of multidrug resistanceassociated protein 4 (MRP4) in pancreatic adenocarcinoma cell lines. PLoS One 10: e0120651.

Cheung L, Flemming CL, Watt F, Masada N, Yu DMT, Huynh T, Conseil G, Tivnan A, Polinsky A, Gudkov AV, et al. (2014) High-throughput screening identifies 
Ceefourin 1 and Ceefourin 2 as highly selective inhibitors of multidrug resistance protein 4 (MRP4). Biochem Pharmacol 91:97-108.

Choi C-H (2005) ABC transporters as multidrug resistance mechanisms and the development of chemosensitizers for their reversal. Cancer Cell Int 5:30.

Conroy T, Desseigne F, Ychou M, Bouché O, Guimbaud R, Bécouarn Y, Adenis A, Raoul J-L, Gourgou-Bourgade S, de la Fouchardière C, et al.; Groupe Tumeurs Digestives of Unicancer; PRODIGE Intergroup (2011) FOLFIRINOX versus gemcitabine for metastatic pancreatic cancer. $N$ Engl J Med 364:1817-1825.

Copsel S, Bruzzone A, May M, Beyrath J, Wargon V, Cany J, Russel FG, Shayo C, and Davio C (2014) Multidrug resistance protein 4/ ATP binding cassette transporter 4: a new potential therapeutic target for acute myeloid leukemia. Oncotarget 5:9308-9321.

Copsel S, Garcia C, Diez F, Vermeulem M, Baldi A, Bianciotti LG, Russel FGM, Shayo C, and Davio C (2011) Multidrug resistance protein 4 (MRP4/ABCC4) regulates cAMP cellular levels and controls human leukemia cell proliferation and differentiation. J Biol Chem 286:6979-6988.

Davio CA, Cricco GP, Bergoc RM, and Rivera ES (1995) H1 and H2 histamine receptors in N-nitroso-N-methylurea (NMU)-induced carcinomas with atypical coupling to signal transducers. Biochem Pharmacol 50:91-96.

Decouture B, Dreano E, Belleville-Rolland T, Kuci O, Dizier B, Bazaa A, Coqueran B, Lompre A-M, Denis CV, Hulot J-S, et al. (2015) Impaired platelet activation and cAMP homeostasis in MRP4-deficient mice. Blood 126:1823-1830.

Dou AX and Wang X (2010) Cyclic adenosine monophosphate signal pathway in targeted therapy of lymphoma. Chin Med J (Engl) 123:95-99.

Fajardo AM, Piazza GA, and Tinsley HN (2014) The role of cyclic nucleotide signaling pathways in cancer: targets for prevention and treatment. Cancers (Basel) 6: 436-458.

Faul F, Erdfelder E, Buchner A, and Lang A-G (2009) Statistical power analyses using $\mathrm{G}^{*}$ Power 3.1: tests for correlation and regression analyses. Behav Res Methods 41:1149-1160.

Faul F, Erdfelder E, Lang A-G, and Buchner A (2007) G*Power 3: a flexible statistical power analysis program for the social, behavioral, and biomedical sciences. Behav Res Methods 39:175-191.

Furukawa T, Kuboki Y, Tanji E, Yoshida S, Hatori T, Yamamoto M, Shibata N, Shimizu K, Kamatani N, and Shiratori K (2011) Whole-exome sequencing uncovers frequent GNAS mutations in intraductal papillary mucinous neoplasms of the pancreas. Sci Rep 1:161.

Fury MG, Krug LM, Azzoli CG, Sharma S, Kemeny N, Wu N, Kris MG, and Rizvi NA (2006) A phase I clinical pharmacologic study of pralatrexate in combination with probenecid in adults with advanced solid tumors. Cancer Chemother Pharmacol 57: 671-677.

Hagmann W, Jesnowski R, Faissner R, Guo C, and Löhr JM (2009) ATP-binding cassette $\mathrm{C}$ transporters in human pancreatic carcinoma cell lines: upregulation in 5-fluorouracil-resistant cells. Pancreatology 9:136-144.

Hausmann S, Kong B, Michalski C, Erkan M, and Friess H (2014) The role of inflammation in pancreatic cancer. Adv Exp Med Biol 816:129-151.

Hochbaum D, Tanos T, Ribeiro-Neto F, Altschuler D, and Coso OA (2003) Activation of JNK by Epac is independent of its activity as a Rap guanine nucleotide exchanger. J Biol Chem 278:33738-33746.

Hofer AM and Lefkimmiatis K (2007) Extracellular calcium and cAMP: second messengers as "third messengers"? Physiology (Bethesda) 22:320-327.

Iglesias-Bartolome R, Torres D, Marone R, Feng X, Martin D, Simaan M, Chen M Weinstein LS, Taylor SS, Molinolo AA, et al. (2015) Inactivation of a G $\alpha(\mathrm{s})$-PKA tumour suppressor pathway in skin stem cells initiates basal-cell carcinogenesis. Nat Cell Biol 17:793-803.

Iwamura T and Hollingsworth MA (2002) Pancreatic tumors, in Human Cell Culture (Masters JRW and Palsson B eds) pp 107-122, Springer, Dordrecht, The Netherlands.

Kanda M, Matthaei H, Wu J, Hong S-M, Yu J, Borges M, Hruban RH, Maitra A, Kinzler K, Vogelstein B, et al. (2012) Presence of somatic mutations in most early-stage pancreatic intraepithelial neoplasia. Gastroenterology 142: 730-733.e9.

Keppler D (2011) Multidrug resistance proteins (MRPs, ABCCs): importance for pathophysiology and drug therapy. Handb Exp Pharmacol 201: 299-323.

Klarenbeek J, Goedhart J, van Batenburg A, Groenewald D, and Jalink K (2015) Fourth-generation epac-based FRET sensors for cAMP feature exceptional brightness, photostability and dynamic range: characterization of dedicated sensors for FLIM, for ratiometry and with high affinity. PLoS One 10:e0122513.

Klein DM and Loizzi RF (1977) Enhancement of R3230AC rat mammary tumor growth and cellular differentiation by dibutyryl cyclic adenosine monophosphate. $J$ Natl Cancer Inst 58:813-818.

Leggas M, Adachi M, Scheffer GL, Sun D, Wielinga P, Du G, Mercer KE, Zhuang Y, Panetta JC, Johnston B, et al. (2004) Mrp4 confers resistance to topotecan and protects the brain from chemotherapy. Mol Cell Biol 24:7612-7621.

Lin ZP, Zhu YL, Johnson DR, Rice KP, Nottoli T, Hains BC, McGrath J, Waxman SG, and Sartorelli AC (2008) Disruption of cAMP and prostaglandin E2 transport by multidrug resistance protein 4 deficiency alters cAMP-mediated signaling and nociceptive response. Mol Pharmacol 73:243-251.

Liu C, Janke LJ, Yang JJ, Evans WE, Schuetz JD, and Relling MV (2017) Differential effects of thiopurine methyltransferase (TPMT) and multidrug resistanceassociated protein gene 4 (MRP4) on mercaptopurine toxicity. Cancer Chemother Pharmacol 80:287-293.

Long J, Zhang Y, Yu X, Yang J, LeBrun DG, Chen C, Yao Q, and Li M (2011) Overcoming drug resistance in pancreatic cancer. Expert Opin Ther Targets 15 817-828.

Lorenz R, Aleksic T, Wagner M, Adler G, and Weber CK (2008) The cAMP/Epac1/ Rap1 pathway in pancreatic carcinoma. Pancreas 37:102-103.
Middleton G, Silcocks P, Cox T, Valle J, Wadsley J, Propper D, Coxon F, Ross P, Madhusudan S, Roques T, et al. (2014) Gemcitabine and capecitabine with or without telomerase peptide vaccine GV1001 in patients with locally advanced or metastatic pancreatic cancer (TeloVac): an open-label, randomised, phase 3 trial. Lancet Oncol 15:829-840.

Miranda ER, Nam EA, Kuspa A, and Shaulsky G (2015) The ABC transporter, AbcB3, mediates cAMP export in D. discoideum development. Dev Biol 397:203-211.

Moitra K (2015) Overcoming multidrug resistance in cancer stem cells. BioMed Res Int 2015:635745.

Moreno MJ, Ball M, Andrade MF, McDermid A, and Stanimirovic DB (2006) Insulinlike growth factor binding protein-4 (IGFBP-4) is a novel anti-angiogenic and antitumorigenic mediator secreted by dibutyryl cyclic AMP (dB-cAMP)-differentiated glioblastoma cells. Glia 53:845-857.

Morgan JA, Cheepala SB, Wang Y, Neale G, Adachi M, Nachagari D, Leggas M, Zhao W, Boyd K, Venkataramanan R, et al. (2012) Deregulated hepatic metabolism exacerbates impaired testosterone production in Mrp4-deficient mice. J Biol Chem 287:14456-14466.

Murray J, Valli E, Yu DMT, Truong AM, Gifford AJ, Eden GL, Gamble LD, Hanssen KM, Flemming CL, Tan A, et al. (2017) Suppression of the ATP-binding cassette transporter ABCC4 impairs neuroblastoma tumour growth and sensitises to irinotecan in vivo. Eur J Cancer 83:132-141.

Naviglio S, Caraglia M, Abbruzzese A, Chiosi E, Di Gesto D, Marra M, Romano M, Sorrentino A, Sorvillo L, Spina A, et al. (2009) Protein kinase A as a biological target in cancer therapy. Expert Opin Ther Targets 13:83-92.

Oevermann L, Scheitz J, Starke K, Köck K, Kiefer T, Dölken G, Niessen J, Greinacher A, Siegmund W, Zygmunt M, et al. (2009) Hematopoietic stem cell differentiation affects expression and function of MRP4 (ABCC4), a transport protein for signaling molecules and drugs. Int J Cancer 124:2303-2311.

O’Hayre M, Vázquez-Prado J, Kufareva I, Stawiski EW, Handel TM, Seshagiri S, and Gutkind JS (2013) The emerging mutational landscape of G proteins and G-protein-coupled receptors in cancer. Nat Rev Cancer 13:412-424.

Prasad KN, Cole WC, Yan X-D, Nahreini P, Kumar B, Hanson A, and Prasad JE (2003) Defects in cAMP-pathway may initiate carcinogenesis in dividing nerve cells: a review. Apoptosis 8:579-586.

Prasad KN and Kumar S (1975) Role of cyclic AMP in differentiation of human neuroblastoma cells in culture. Cancer 36:1338-1343.

Propper DJ, Saunders MP, Salisbury AJ, Long L, O’Byrne KJ, Braybrooke JP, Dowsett M, Taylor M, Talbot DC, Ganesan TS, et al. (1999) Phase I study of the novel cyclic AMP (cAMP) analogue 8-chloro-cAMP in patients with cancer: toxicity, hormonal, and immunological effects. Clin Cancer Res 5: 1682-1689.

Rahib L, Smith BD, Aizenberg R, Rosenzweig AB, Fleshman JM, and Matrisian LM (2014) Projecting cancer incidence and deaths to 2030: the unexpected burden of thyroid, liver, and pancreas cancers in the United States. Cancer Res 74: 2913-2921.

Rius M, Nies AT, Hummel-Eisenbeiss J, Jedlitschky G, and Keppler D (2003) Cotransport of reduced glutathione with bile salts by MRP4 (ABCC4) localized to the basolateral hepatocyte membrane. Hepatology 38:374-384.

Rodríguez CI, Castro-Pérez E, Prabhakar K, Block L, Longley BJ, Wisinski JA, Kimple ME, and Setaluri V (2017) EPAC-RAP1 axis-mediated switch in the response of primary and metastatic melanoma to cyclic AMP. Mol Cancer Res 15: 1792-1802.

Russel FGM, Koenderink JB, and Masereeuw R (2008) Multidrug resistance protein 4 (MRP4/ABCC4): a versatile efflux transporter for drugs and signalling molecules. Trends Pharmacol Sci 29:200-207.

Sahoo D, Dill DL, Tibshirani R, and Plevritis SK (2007) Extracting binary signals from microarray time-course data. Nucleic Acids Res 35:3705-3712.

Sassi Y, Hara Y, Lompré A-M, and Hulot J-S (2009) Multi-drug resistance protein 4 (MRP4/ABCC4) and cyclic nucleotides signaling pathways. Cell Cycle 8: 962-963.

Sassi Y, Lipskaia L, Vandecasteele G, Nikolaev VO, Hatem SN, Cohen Aubart F, Russel FG, Mougenot N, Vrignaud C, Lechat P, et al. (2008) Multidrug resistanceassociated protein 4 regulates cAMP-dependent signaling pathways and controls human and rat SMC proliferation. J Clin Invest 118:2747-2757.

Savai R, Pullamsetti SS, Banat G-A, Weissmann N, Ghofrani HA, Grimminger F, and Schermuly RT (2010) Targeting cancer with phosphodiesterase inhibitors. Expert Opin Investig Drugs 19:117-131.

Scavennec J, Carcassonne Y, Gastaut JA, Blanc A, and Cailla HL (1981) Relationship between the levels of cyclic cytidine $3^{\prime}: 5^{\prime}$-monophosphate, cyclic guanosine $3^{\prime}: 5^{\prime}$ monophosphate, and cyclic adenosine $3^{\prime}: 5^{\prime}$-monophosphate in urines and leukocytes and the type of human leukemias. Cancer Res 41:3222-3227.

Schaap P (2011) Evolutionary crossroads in developmental biology: Dictyostelium discoideum. Development 138:387-396.

Schindelin J, Arganda-Carreras I, Frise E, Kaynig V, Longair M, Pietzsch T, Preibisch S, Rueden C, Saalfeld S, Schmid B, et al. (2012) Fiji: an open-source platform for biological-image analysis. Nat Methods 9:676-682.

Schneider CA, Rasband WS, and Eliceiri KW (2012) NIH Image to ImageJ: 25 years of image analysis. Nat Methods 9:671-675.

Schwede F, Maronde E, Genieser H, and Jastorff B (2000) Cyclic nucleotide analogs as biochemical tools and prospective drugs. Pharmacol Ther 87: $199-226$

Selen A, Amidon GL, and Welling PG (1982) Pharmacokinetics of probenecid following oral doses to human volunteers. J Pharm Sci 71:1238-1242.

Siegel RL, Miller KD, and Jemal A (2017) Cancer statistics, 2017. CA Cancer J Clin 67:7-30.

Stork PJS and Schmitt JM (2002) Crosstalk between cAMP and MAP kinase signaling in the regulation of cell proliferation. Trends Cell Biol 12:258-266.

Takenaka K, Morgan JA, Scheffer GL, Adachi M, Stewart CF, Sun D, Leggas M, Ejendal KFK, Hrycyna CA, and Schuetz JD (2007) Substrate overlap between 
Mrp4 and Abcg2/Bcrp affects purine analogue drug cytotoxicity and tissue distribution. Cancer Res 67:6965-6972.

Therneau TM and Grambsch PM (2000) Modeling Survival Data: Extending the Cox Model, Springer, New York.

Tian Q, Zhang J, Chan SY, Tan TMC, Duan W, Huang M, Zhu Y-Z, Chan E, Yu Q, Nie $\mathrm{Y}-\mathrm{Q}$, et al. (2006) Topotecan is a substrate for multidrug resistance associated protein 4. Curr Drug Metab 7:105-118.

Van Aubel RA, Smeets PH, van den Heuvel JJ, and Russel FG (2005) Human organic anion transporter MRP4 (ABCC4) is an efflux pump for the purine end metabolite urate with multiple allosteric substrate binding sites. Am J Physiol Renal Physiol 288:327-333.

Vitali E, Cambiaghi V, Spada A, Tresoldi A, Zerbi A, Peverelli E, Carnaghi C, Mantovani G, and Lania AG (2015) cAMP effects in neuroendocrine tumors: the role of Epac and PKA in cell proliferation and adhesion. Exp Cell Res 339:241-251.

Von Hoff DD, Ervin T, Arena FP, Chiorean EG, Infante J, Moore M, Seay T, Tjulandin SA, Ma WW, Saleh MN, et al. (2013) Increased survival in pancreatic cancer with nab-paclitaxel plus gemcitabine. $N$ Engl J Med 369: 1691-1703.

Warrier S, Pavanram P, Raina D, and Arvind M (2012) Study of chemoresistant CD133+ cancer stem cells from human glioblastoma cell line U138MG using multiple assays. Cell Biol Int 36:1137-1143.

Wen J, Luo J, Huang W, Tang J, Zhou H, and Zhang W (2015) The pharmacological and physiological role of multidrug-resistant protein 4. J Pharmacol Exp Ther $\mathbf{3 5 4}$ $358-375$.
Wu J, Matthaei H, Maitra A, Dal Molin M, Wood LD, Eshleman JR, Goggins M, Canto MI, Schulick RD, Edil BH, et al. (2011) Recurrent GNAS mutations define an unexpected pathway for pancreatic cyst development. Sci Transl Med 3:92ra66.

Xie M, Rich TC, Scheitrum C, Conti M, and Richter W (2011) Inactivation of multidrug resistance proteins disrupts both cellular extrusion and intracellular degradation of cAMP. Mol Pharmacol 80:281-293.

Yaneff A, Sahores A, Gomez N, Carozzo A, Shayo C, and Davio C (2017) MRP4/ABCC4 as a new therapeutic target: meta-analysis to determine cAMP binding sites as a tool for drug design. Curr Med Chem DOI: 10.2174/0929867325666171229133259 [published ahead of print].

Zamek-Gliszczynski MJ, Nezasa K, Tian X, Bridges AS, Lee K, Belinsky MG, Kruh GD, and Brouwer KLR (2006) Evaluation of the role of multidrug resistanceassociated protein (Mrp) 3 and Mrp4 in hepatic basolateral excretion of sulfate and glucuronide metabolites of acetaminophen, 4-methylumbelliferone, and harmol in Abcc3-/- and Abcc4-/- mice. J Pharmacol Exp Ther 319:1485-1491.

Zhang Z, Wang J, Shen B, Peng C, and Zheng M (2012) The ABCC4 gene is a promising target for pancreatic cancer therapy. Gene 491:194-199.

Address correspondence to: Dr. Carlos Davio, Instituto de Investigaciones Farmacológicas (ININFA-UBA-CONICET), Facultad de Farmacia y Bioquímica, Universidad de Buenos Aires, Junín 956 (1113), Ciudad Autónoma de Buenos Aires, Capital Federal 1113, Argentina. E-mail: cardavio@ffyb.uba.ar 